\title{
Lean premixed opposed jet flames in fractal grid generated multiscale turbulence
}

\author{
K. H. H. Goh, P. Geipel, R. P. Lindstedt* \\ Department of Mechanical Engineering, Imperial College, \\ Exhibition Road, London SW7 2AZ, UK
}

\begin{abstract}
The opposed jet configuration presents an attractive canonical geometry for the evaluation of burning properties of turbulent flames with past studies typically limited to low Reynolds numbers. Fractal grid generated turbulence was used to remove the low turbulence level limitations associated with conventional perforated plate generators with the turbulent Reynolds number range moved from 50-120 to 130-318. Optimal grid configurations were determined with particular emphasis on reducing the impact of the flow upstream of the turbulence generators in order to facilitate simpler boundary conditions for computational studies. The resulting flow structures were analysed using proper orthogonal decomposition and conditional proper orthogonal decomposition. Velocity and reaction progress variable statistics, including conditional velocities and scalar fluxes, are reported for fuel lean methane, ethylene and propane flames approaching extinction. The instrumentation comprised particle image velocimetry with the flows to both nozzles seeded with $1 \mu \mathrm{m}$ silicon oil droplets or $3 \mu \mathrm{m} \mathrm{Al}_{2} \mathrm{O}_{3}$ particles. Probability density functions were determined for the instantaneous location of the stagnation point to eliminate the possibility of low frequency bulk motion distorting velocity statistics. Probability density functions of flame curvature were determined using a multi-step flame front detection algorithm with estimates of the turbulent burning velocity provided along with a discussion of alternative determination methods. The data sets show that fractal grids generate multi-scale broadband turbulence and present an opportunity for a systematic evaluation of calculation methods for premixed turbulent flames that undergo a transition from non-gradient to gradient turbulent transport while approaching extinction.
\end{abstract}

Keywords: Opposed Jets, Fractal Grids, Premixed Flames, Multiscale Turbulence, Conditional Statistics

\footnotetext{
${ }^{*}$ Corresponding author. Fax: +44 2075945696.

Email address: p.lindstedt@imperial.ac.uk (R. P. Lindstedt)
} 


\section{Introduction}

Opposed jet geometries have been used extensively to investigate premixed and non-premixed combustion under laminar and turbulent flow conditions. The comparatively simple flame stabilisation method and essentially adiabatic conditions lead to flame dynamics and extinction being related to the aerothermochemistry of the combustion process rather than heat losses. Combined with the comparatively simple boundary conditions, the opposed jet geometry provides an attractive standard test case for the assessment of fuel effects and closure approximations as proposed by Bray et al. [1], Lindstedt and Váos [2, 3] and subsequently by Geyer et al. [4, 5] in the context of large eddy simulations (LES). Preparatory isothermal flow studies have also been presented by a number of investigators (e.g. Geipel et al. [6], Kostiuk et al. [7], Korusoy and Whitelaw [8], Lindstedt et al. [9]) and flame structure studies include velocity and scalar field measurements by Mounaïm-Rouselle and Gökalp [10], Kostiuk et al. [11] and Lindstedt et al. [12]. Strain effects and extinction and relight characteristics were discussed by Kostiuk et al. [13], Mounaïm-Rouselle and Gökalp [14], Sardi and Whitelaw [15], Korusoy and Whitelaw [16] and Luff et al. [17] amongst others. Mounaïm-Rouselle and Gökalp [10] and Kostiuk et al. [13, 18] showed that larger nozzle separations caused a low frequency axial movement made visible by a bouncing of the flame brush. The stabilisation of the flame brush off the symmetry plane of the burner was also noticeable. It was suggested that an interaction of the jets with the surrounding air caused both phenomena and difficulties were reduced with the introduction of co-flowing streams as confirmed by a more homogeneous turbulence distribution with less energy at lower frequencies. Subsequent studies (e.g. [12, 17]) have 
shown that the flow symmetry can be much improved for nozzle separations $\mathrm{H} / \mathrm{D} \simeq 1$, where $\mathrm{H}$ is the nozzle separation and $\mathrm{D}$ the nozzle diameter, with low frequency instabilities removed and overall improved stability [6].

The comparatively low turbulence levels achieved in earlier studies at the point of flame extinction have proved problematic for computational methods based on high Reynolds number assumptions. The same observation [9] also applies for isothermal flows at Reynolds (Re) numbers below 10,000 and higher values were required in order to provide good agreement with numerical simulations when perforated plates were used as turbulence generators. However, such conditions were found to lead to early extinction for flames featuring alkane fuels due to the presence of bulk strain at nozzle separations small enough $(\mathrm{H} / \mathrm{D} \leq 1)$ to prevent axial bulk instabilities in the flow. Coppola and Gomez [19] used high blockage ratio turbulence generators to increase turbulence levels substantially and suggested [20] the use of the geometry as a benchmark for practical systems. The turbulence generators were subsequently found trigger large scale oscillations of the stagnation surface at frequencies $<200 \mathrm{~Hz}$ and it was suggested that such instabilities need to be screened, e.g. using Proper Orthogonal Decomposition (POD), to eliminate artifacts (e.g. in turbulence levels) from the measurements [21]. However, it has subsequently been suggested [22] that flows with combustion require conditional POD (CPOD) as conventional POD does not distinguish between fluid structures in reactants and products. The filtering of instabilities using the latter approach can hence arguably only be applied to flows without significant density variations. Fractal grids are here used to avoid such difficulties and to provide significantly increased turbulence levels without any issues arising with respect to bulk flow instabilities. The use of fractal grids was first presented in the context of wind tunnels by 
Vassilicos et al. [23, 24] and later by Hurst et al. [25]. The first application of fractal grids in an opposed jet geometry was presented by Geipel et al. [6] in a study of isothermal flows. It was shown that such grids can increase turbulent Reynolds $\left(R e_{t}\right)$ numbers in excess of a factor of two for the same bulk velocity as compared to conventional perforated plate turbulence generators. It has also been shown that fractal grids can substantially enhance turbulent diffusion $[26,27]$. The consequence is a significant change in the balance of turbulent to bulk strain while also maintaining acceptable flow symmetry [6]. The fractal grid approach was subsequently used by Goh et al. [28] to explore the transition of premixed JP-10 flames from the corrugated flamelet regime to a Homogeneous Charge Diffusion Ignition (HCDI) [29] related flameless combustion mode and by Goh et al. [22] to analyse conventional opposed jet flames.

The current work extends past studies of opposed jet flames by: (i) The use of multiscale fractal grid generated turbulence in a substantially revised configuration aimed at providing increased turbulent strain while providing simplified upstream boundary conditions in order to facilitate computational studies. The turbulent Reynolds number range is moved from 50-120 to 130318, as compared to conventional perforated plate generators. (ii) Velocity and reaction progress variable statistics, including conditional velocities and scalar fluxes, are reported for stoichiometric and fuel lean methane, ethylene and propane flames approaching extinction. (iii) The turbulence structure obtained using conventional and fractal grids was analysed using POD and CPOD [22] techniques. (iv) Probability density functions were determined for the instantaneous location of the stagnation point and show the movement to be of the order of the integral length scale and hence not influenced by unstable bulk flow motion. (v) Probability density functions of flame 
curvature were determined using a validated multi-step flame front detection algorithm $[22,28]$. (vi) Estimates of turbulent burning velocities are provided and differences associated with alternative determination methods quantified. Finally, (vii) the rate of dissipation was estimated using the velocity gradient based technique of George and Hussein [30]. Overall, the data sets present an opportunity for a systematic evaluation of calculation methods for premixed turbulent flames approaching extinction.

\section{Experimental configuration, techniques and uncertainties}

The opposed jet geometry used as a starting point in the current work consists of two nozzles in a vertical arrangement originally designed by Geyer et al. $[5,31]$ and is identical to that described by Geipel et al. [6]. Both nozzles were water-cooled to prevent differences in the reactant densities due to preheating. The maximum positional uncertainties for the nozzles with the current configuration are $0.2 \mathrm{~mm}$ for the coaxial alignment and $0.5^{\circ}$ in the angular alignment. The outlet of each nozzle is $30 \mathrm{~mm}$ in diameter and, for the base case configuration, turbulence is generated $50 \mathrm{~mm}$ upstream of the nozzle exit plane using perforated plates with a hole diameter of $4 \mathrm{~mm}$ and a blockage of $45 \%$ as shown in Fig. 1 . The perforated plates are similar to those used by Mastorakos et al. [32-34], Sardi et al. [15, 35, 36] and Lindstedt

et al. [9, 12]. Fractal grids [6, 23-25] subsequently replaced the perforated plates and were used to increase turbulence intensities at the nozzle exits. Following an extensive experimental study of the corresponding isothermal flow field [6], a space-filling fractal cross grid with a total blockage of $65 \%$ and fractal dimensions corresponding to a maximum bar width of $2.0 \mathrm{~mm}$ and a minimum width of $0.5 \mathrm{~mm}$ was chosen as shown in Fig. 1. The selected 
fractal grid increased the turbulent Reynolds number range from 50-120 to 130-318 for the same bulk velocity range of $4.0 \mathrm{~m} / \mathrm{s}$ to $8.0 \mathrm{~m} / \mathrm{s}$, based on the integral length scale $\left(l_{t}\right)$ of $\simeq 3.1 \mathrm{~mm}$ determined by Geipel et al. [6], with turbulence levels measured at the nozzle exits. Goh [37] determined the corresponding integral length scales for the current revised geometry, discussed below, and obtained marginally higher values of $\simeq 3.5 \mathrm{~mm}$ at $4.0 \mathrm{~m} / \mathrm{s}$ bulk velocity and $\simeq 3.2 \mathrm{~mm}$ at $8.0 \mathrm{~m} / \mathrm{s}$. The estimation of length scales and the impact on the fitting of longitudinal energy spectra based on hot wire anemometry and PIV data has been discussed by Geipel et al. [6]. The axial instability of the flow observed at higher nozzle separations (e.g. $[13,17,18])$ was effectively removed by the selected H/D (=1) ratio and further reduced by the presence of a co-flow [10]. The co-flowing stream of air was set to a velocity of $\geq 10 \%$ of the corresponding bulk flow velocity of the reactant mixture in order to reduce the effect of the shear layer that forms between the opposed jet flows and the ambient air.

Dry and filtered air was supplied to each nozzle at 4 bar(g) by a compressor using digital mass flow controllers (Bronkhorst UK LTD). The deviation of each mass flow controller was $\leq 0.8 \%$ RD (reading) plus $\leq 0.2 \%$ FS (full scale) and the reproducibility better than $\leq 0.1 \%$. Two additional mass flow controllers, each calibrated for methane, propane and ethylene, were used to control the fuel flows. The fuels were supplied using bottles of either methane (99\% purity), propane (96\% purity) and ethylene (99.9\% purity) at $3.0 \mathrm{bar}(\mathrm{g})$. The mass flow rates were monitored using a custom written LabView interface connected to a Readout/Control unit Type E-7000 that was connected via a flow-bus system to each of the four mass flow controllers. The mixing of the reactants followed the approach described by Lindstedt et al. [12] with overall bulk velocities in the range $4.0 \mathrm{~m} / \mathrm{s}$ to 
$8.0 \mathrm{~m} / \mathrm{s}$.

A Particle Image Velocimetry (PIV) system (LaVision Flowmaster 3) was used to measure the axial and radial velocity components. The central plane perpendicular to the nozzle exits was illuminated by two $120 \mathrm{~mJ}$ SoloNew Wave double pulse Nd:YAG lasers. The laser head was equipped with LaVision light sheet optics that allowed an adjustable light sheet thickness between $0.5 \mathrm{~mm}$ and $2.5 \mathrm{~mm}$. The thickness was set to $\simeq 0.8 \mathrm{~mm}$ for all measurements presented. The flow field was viewed using a 12 bit, 1376x1040 pixel, $10 \mathrm{~Hz}$ CCD Imager Intense camera equipped with a $50 \mathrm{~mm}$ Nikon lens. The time between the two PIV-images was adjusted between 15-40 $\mu \mathrm{s}$ according to the mean bulk velocity in order to reduce spurious vectors. For each correlation, two images were divided into smaller interrogation windows and a decreasing window size starting from 128x128, via 64x64 down to $32 \times 32$ pixels was chosen with a $50 \%$ overlap leading to a vector spacing of $0.4 \mathrm{~mm}$. Høst-Madsen and Nielsen [38] estimated that an interrogation window size of $0.2 l_{t}$ would result in approximately a $10 \%$ uncertainty in the fluctuations. The current integral length scales result in an estimated uncertainty in the turbulent fluctuations of $\leq 15 \%$. All scalar and velocity data was obtained by averaging at least 1000 statistically independent instantaneous vector fields using a purpose written FORTRAN program. Further post-processing featured a window based algorithm for the determination of the instantaneous stagnation point location. The window size was set to 36 times the vector spacing $(0.4 \mathrm{~mm})$. The rotation of the instantaneous stagnation plane was determined by applying a linear fit to the two outermost points of the stagnation point streamlines.

Silicon oil droplets with an average diameter of $1 \mu \mathrm{m}$ produced by two PALAS Aerosol Generators Type AGF 10.0 were used to determine the reac- 
tion progress variable statistics and conditional reactant velocities. Velocity data was also obtained using aluminium-oxide particles with an average diameter of 3-5 $\mu \mathrm{m}$ as measured by a Cilas 1064 particle size analyser. The number of excited pixels on the CCD-image was kept between two and five. This is in keeping with the optimal particle image size and therefore avoided errors associated with peak-locking, described in greater detail by Angele and Muhammad-Klingmann [39]. Furthermore, the RMS-uncertainty was kept at a minimum by using a particle image diameter of more than one pixel [40]. Other suggestions for the optimal particle image diameter range from 1.5 pixels [41], 2.0 pixels [42] and up to 4.0 pixels [43]. The latter value was obtained by a Monte-Carlo calculation including velocity gradients due to shear. The authors suggested that the presence of shear was the reason for the increase of the optimal particle size on the PIV-images.

Stagnating flows have a high dynamic range with the minimum velocity approaching zero at the stagnation plane where the largest errors can be expected [12]. The relaxation time of a seeding particle to a step change in the fluid velocity was estimated using the approach of Han and Mungal [44].

$$
\tau_{s}=d_{p}^{2} \frac{\rho_{p}}{18 \mu}
$$

The relaxation time for the $d_{p}=1 \mu \mathrm{m}$ silicon oil droplets $\left(\rho_{p}=980 \mathrm{~kg} / \mathrm{m}^{3}\right)$ was estimated at $\simeq 3 \mu \mathrm{s}$ and for the $d_{p}=3 \mu \mathrm{m} \mathrm{Al}{ }_{2} \mathrm{O}_{3}$ particles $\left(\rho_{p}=3,900\right.$ $\left.\mathrm{kg} / \mathrm{m}^{3}\right)$ the relaxation time was $\simeq 29 \mu \mathrm{s}$ in the products $\left(\mu=6.759410^{-5}\right.$ $\mathrm{kg} / \mathrm{m} / \mathrm{s})$ and $\simeq 109 \mu \mathrm{s}$ in the reactants $\left(\mu=1.783510^{-5} \mathrm{~kg} / \mathrm{m} / \mathrm{s}\right)$ [45]. The smallest PIV scale based on the largest bulk velocity of $8.0 \mathrm{~m} / \mathrm{s}$ and the smallest interrogation window size (32x32 pixels) is around $50 \mu$ s and sufficiently large for particles to follow the flow in the products. Particle lag in the reactants could impact the $\mathrm{Al}_{2} \mathrm{O}_{3}$ seeding though previous work [12] 
has shown that the reactant velocities obtained for both types of seeding were within $15 \%$.

Optical measurement techniques that rely on seeding particles (e.g. LDV and PIV) can suffer from effects of thermophoresis leading to a particle velocity lag as shown by Sung et al. [46, 47]. Gomez and Rosner [48] suggest a local particle drift velocity $\left(v_{T}\right)$ as shown Eq. (2), which was approximated by Amantini et al. [49] using Eq. (3), where $\alpha_{T}$ is the dimensionless thermophoretic diffusion factor, $\mathrm{D}$ the particle Brownian diffusivity, $\mathrm{T}$ the temperature, $\alpha_{m}$ the tangential momentum accommodation coefficient between the fluid and the solid particle (often assumed to be unity) and $\nu$ the kinematic viscosity.

$$
\begin{gathered}
v_{T}=\left(\alpha_{T} D\right)_{\text {particle }} \frac{-\nabla T}{T} \\
v_{T} \simeq 0.75\left[1+\frac{\pi}{8} \alpha_{m}\right] \nu \frac{-\nabla T}{T}
\end{gathered}
$$

In the vicinity of the flame front the effect of thermophoresis might be greater than the uncertainty in the PIV data in which case the effect needs to be considered as shown by Amantini et al. [49], Chelliah et al. [50] and Sung et al. [46]. For an ethylene flame $(\phi=0.9)$ it was estimated that $v_{T} \simeq 0.49 \mathrm{~m} / \mathrm{s}$ based on premixed laminar flame data. The resulting uncertainty in velocity statistics across the flame front is $\leq 20 \%$ when evaluated relative to the corresponding bulk velocity. For other flames the uncertainty was $\leq 10 \%$.

Beam steering effects caused by a change in the refractive index of the fluid and the impact of the density gradient across a flame front were quantified by Kaiser et al. [51] using a ray-tracing technique based on Rayleigh images as an approximate measure of the index-of-refraction field. The study indicated that the spread of the rays in the image plane was on the sub-pixel scale and therefore negligible as also discussed by Frank et al. [52]. 
Böhm [53] found, by comparing time--correlated OH-LIF images obtained in an opposed jet geometry, that the flame movement between two images $50 \mu \mathrm{s}$ apart was negligible and hence the uncertainty of the velocity measurements caused by beam steering in the measured time interval could be ignored. The time interval between the PIV images in the current work was between $15-40 \mu$ s and uncertainties caused by beam steering were neglected.

\subsection{Flame front detection}

The reaction progress variable statistics were produced from PIV images using a custom written density segregation algorithm, described by Goh et al. [22, 28], to identify gradients created by changes in seeding density due to heat release. Similar algorithms have been developed and shown capable of producing excellent agreement with flame fronts obtained using simultaneous CH-LIF/PIV by Steinberg et al. [54] or $\mathrm{OH} / \mathrm{CH}_{2} \mathrm{O}-\mathrm{LIF} / \mathrm{PIV}$ by Pfadler et al. [55]. The current algorithm was initially validated [22] using images of a silicon oil seeding that evaporates as it passes through the flame front as previously discussed for stagnating flows by Shepherd et al. [57], Kostiuk et al. [13] and Lindstedt et al. [12]. The technique was further validated by Goh et al. [28] in the presence of reaction zone broadening by comparing with OH-PLIF images obtained using the $\mathrm{Ba}\left(\mathrm{NO}_{3}\right)_{2}$ crystal based technique of Kerl et al. [56]. The first step in the algorithm is to determine the mean intensity of the Mie-scattering in a defined reference window. Goh et al. [22] have discussed the thresholding process in detail and inspection of particle images suggest a Gaussian nature. The step naturally filters out background noise should images contain significant noise from reflections, non-uniform laser intensity or laser sheet thickness. The filtering produces binary images with an equal weight given to all accepted peaks. Subsequently, using 
the density of peaks in the reference region, gaps between peaks were filled using a moving average filter to avoid leaving gaps in the reactant regions due to minor fluctuations in seeding densities. Subsequently, a bilateral filter [58] was used to reduce the intensity fluctuations in the reactant and product regions while preserving the steep gradients at the flame front. The latter was achieved by using convolution of range and distance filters with the image produced by the moving average filter. The resulting image had a relatively smooth histogram with multiple peaks representing segmented regions of similar intensities. By applying a cubic spline fit to the histogram of the resulting image, as applied by Pfadler et al. [59] to filtered images, the nearest minima of the intensity lower than the minimum intensity in the reference window was used as a threshold for creating a binary image. Canny edge detection [60] with a Sobel operator was then applied on the resulting binary image to obtain steep gradients. Subsequently, non-maxima suppression, followed by a skeleton generating algorithm [61], were used to thin the resulting edges to a thickness of one pixel with the flame front identified as the longest line closest to the reference window. The process avoided the identification of the flame front from the opposing reactant stream should seeding densities in both reference windows be the same.

Two data sets, obtained with $\mathrm{Al}_{2} \mathrm{O}_{3}$ or silicon oil seeding with each containing 1000 PIV images, were obtained for identical flow conditions. Examples of images are shown in Fig. 3 where each pixel is around $0.025 \mathrm{~mm}$ in size. Silicon oil evaporates across the instantaneous flame front resulting in a binary PIV image without further treatment. The developed algorithm was applied to both sets of data and the first two moments of the reaction progress variable statistics showed only subtle differences as exemplified in Fig. 4 for methane/air flames at $\phi=0.90$. The slight asymmetry observed 
is of the order $0.4 \mathrm{~mm}$ and close to the estimated Kolmogorov scale in the combustion products. The mean progress variable was marginally lower for aluminium oxide in the vicinity of the stagnation point. However, the effect is arguably due to the greater heat losses caused by the aluminium oxide seeding which results in lower flame temperatures and a modest decrease in the distance between the twin flames. The current silicon oil evaporates at $550 \mathrm{~K}$ and the detected flame fronts will be biased towards the reactant streams. The discrepancies are of the order of $0.5 \mathrm{~mm}$, which is comparable to the laminar flame thickness as determined by simulations featuring detailed chemistry [45]. Flame curvature statistics were also calculated to provide a further example. The $\mathrm{x}(\mathrm{s})$ and $\mathrm{y}(\mathrm{s})$ coordinates, where s denotes the distance along the curve, were fitted as close to the normalised curve as possible using cubic splines. Subsequently, the curvature h(s) was calculated using Eq. (4) [66].

$$
h(s)=\frac{\frac{d x}{d s} \frac{d^{2} y}{d s^{2}}-\frac{d^{2} x}{d s^{2}} \frac{d y}{d s}}{\left(\left(\frac{d x}{d s}\right)^{2}+\left(\frac{d y}{d s}\right)^{2}\right)^{3 / 2}}
$$

Based on the coordinate system the curvature is positive for upper flame fronts convex to products and for lower flame fronts concave to products. The use of cubic splines was found necessary as traditional (e.g. finite difference) methods created noise in the curvature calculations. The curvature statistics presented here were solely used for verification of the algorithm as precise curvature calculations across a large range of lengthscales remains intractable. The calculated statistics from 500 images in each set are presented in Fig. 5. There is agreement for the curvature pdf for the upper flame, with some discrepancies for the lower flame close to zero curvature. 
The data presented were obtained with a perforated plate without a fitted impact plate and individual frames were investigated to ensure that the effect was not caused by the flame front detection algorithm.

\subsection{Conditional Proper Orthogonal Decomposition}

The instantaneous progress variable $(c)$ was also used to segregate the reactants from the products to obtain unconditional $(\mathrm{u})$ Proper Orthogonal Decomposition (POD) and conditional (CPOD) reactant (r) and product

(p) data using the method of Goh et al. [22] with the spatial mean of the turbulence kinetic energy $(\bar{E})$ obtained using Eqs. (5).

$$
\begin{array}{r}
\bar{E}_{\mathrm{r}}=\sum_{n=1}^{N_{\text {img }}} \sum_{\forall(\mathrm{r}, \mathrm{x})}\left[\frac{1}{2}\left(u_{\mathrm{r}}^{\prime} u_{\mathrm{r}}^{\prime}+v_{\mathrm{r}}^{\prime} v_{\mathrm{r}}^{\prime}\lfloor\lfloor 1-c\rfloor)\right] \div \sum_{n=1}^{N_{\text {img }}} \sum_{\forall(\mathrm{r}, \mathrm{x})}\lfloor 1-c\rfloor\right. \\
\bar{E}_{\mathrm{p}}=\sum_{n=1}^{N_{\text {img }}} \sum_{\forall(\mathrm{r}, \mathrm{x})}\left[\frac{1}{2}\left(u_{\mathrm{p}}^{\prime} u_{\mathrm{p}}^{\prime}+v_{\mathrm{p}}^{\prime} v_{\mathrm{p}}^{\prime}\right)\lfloor c\rfloor\right] \div \sum_{n=1}^{N_{\text {img }}} \sum_{\forall(\mathrm{r}, \mathrm{x})}\lfloor c\rfloor \\
\bar{E}_{\mathrm{u}}=\sum_{n=1}^{N_{\text {img }}} \sum_{\forall(\mathrm{r}, \mathrm{x})}\left[\frac{1}{2}\left(u^{\prime} u^{\prime}+v^{\prime} v^{\prime}\right)\right] \div \sum_{n=1}^{N_{\text {img }}} \sum_{\forall(\mathrm{r}, \mathrm{x})} 1
\end{array}
$$

The spatial mean of the absolute kinetic energy represented by each mode was determined by simply calculating $\bar{E}$, the normalised values $E_{m, \text { norm }}$ and the cumulative energy represented by $M$ modes.

Goh et al. [22] also applied the method of George and Hussein [30] to estimate the rate of turbulence dissipation $(\epsilon)$ from PIV data using a linear combination of mean square values of local velocity gradients and the kinematic velocity $(\nu)$. The Kolmogorov length scale was estimated to be $\simeq 0.1 \mathrm{~mm}$ in the reactants and $\simeq 0.5 \mathrm{~mm}$ in the products under similar conditions. The corresponding Kolmogorov velocities were estimated to be 
$\simeq 0.16 \mathrm{~ms}^{-1}$ in the reactants and $\simeq 0.78 \mathrm{~ms}^{-1}$ in the products. The same method was applied in the current work as discussed below.

\section{Results}

The current study presents data obtained for turbulent premixed opposed jet flames featuring methane, propane and ethylene fuels as well as isothermal flow conditions. The latter were included to provide additional information on the flow at the nozzle exits as well as further information on the influence of the flow path upstream of the turbulence generators on velocity statistics and eigenmodes as determined using POD and CPOD.

The section upstream of the turbulence generators was modified by fitting four $4 \mathrm{~mm}$ or $8 \mathrm{~mm}$ equally spaced inlet jets to explore the impact of flow conditions leading up to the turbulence generators. For the same purpose, additional impact plates were also placed upstream of the turbulence generating plates in order to break up the four inlet jets and to explore the impact on the resulting turbulence intensities at the nozzle exits. The burner geometry is shown in Fig. 2 and the six investigated turbulence generator configurations are summarised below.

- Case 1: Conventional turbulence generator without fitted impact plate and (a) $4 \mathrm{~mm}$ or (b) $8 \mathrm{~mm}$ equally spaced inlet jets.

- Case 2: Conventional turbulence generator with fitted impact plate and (a) $4 \mathrm{~mm}$ or (b) $8 \mathrm{~mm}$ equally spaced inlet jets.

- Case 3: Fractal grid without fitted impact plate and $4 \mathrm{~mm}$ equally spaced inlet jets. 
- Case 4: Fractal grid with fitted impact plate and $4 \mathrm{~mm}$ equally spaced inlet jets.

The above configurations were initially investigated for isothermal flows in preparation for the subsequent studies featuring combustion.

\subsection{Isothermal flows}

The mean axial and radial velocity profiles obtained using traditional perforated plate turbulence generators (Cases 1 and 2) under isothermal flow conditions are presented in Fig. 6 for a location $1.5 \mathrm{~mm}$ downstream of the nozzle exit plane. The current mean velocity profiles show the same trends as presented by Lindstedt et al. [9] and Geyer et al. [31]. A reduction is apparent for all mean axial velocity profiles towards the burner centreline due to the pressure increase along the stagnation point streamline, as also discussed by Korusoy and Whitelaw [8], for all four upstream flow conditions. The use of the additional impact plates (Cases $2 \mathrm{a}$ and $2 \mathrm{~b}$ ) resulted in a stronger velocity gradient towards the nozzle centre for the $4 \mathrm{~mm}$, as compared to the $8 \mathrm{~mm}$, jets. Further comparisons of the nozzle exit profiles show that the impact plates also suppressed the influence of the nozzle inlet velocity and ensured independence of the mean velocity exit profiles of conditions further upstream. However, axial and radial velocity fluctuations across the nozzle exits exhibit a much greater dependency on the flow path upstream of the turbulence generators as shown in Fig. 7. The use of $4 \mathrm{~mm}$ inlet jets without impact plates provided turbulence fluctuations up to $20 \%$ of the corresponding bulk velocity $\left(U_{b}\right)$ as compared to values around $9 \%$ with impact plates fitted. The latter value is in agreement with data presented in earlier studies $[6,9]$. An integral length scale of $2.6 \mathrm{~mm}$ for the perforated plates [12] results in a turbulent Reynolds number of approximately 50 for 
the flow using impact plates. The increased velocity fluctuations caused by the removal of the impact plates is solely the result of direct jet impingement on the perforated plates. A reduction of the inlet jet velocity by a factor of four, through the use of $8 \mathrm{~mm}$ jets, resulted in a 100\% decrease in the velocity fluctuations across the nozzle diameter. Accordingly, it can be concluded that the conventional perforated plate turbulence generators are strongly dependent upon the upstream flow conditions.

Exchanging the perforated plates with fractal grids provided the expected $\sim 100 \%$ increase in fluctuation levels [6]. The velocity statistics $1.5 \mathrm{~mm}$ downstream of the nozzle exits are presented in Fig. 8 for Cases 3 and 4 . Due to (small) uncertainties in the manufacturing process of the fractal grid structure, it cannot be assumed a priori that the nozzle exit profiles are completely symmetric in the circumferential direction. Therefore, velocity statistics for each flow condition were obtained using four separate measurements each with a $45^{\circ}$ rotation in the circumferential direction and the error bars shown in each graph indicate the maximum deviations obtained for the four data sets. Differently from the conventional grids, it is apparent that the introduction of impact plates does not cause a significant reduction in turbulence intensities. Comparisons of the velocity statistics obtained using $4 \mathrm{~mm}$ inlet jets with and without the additional impact plates combined with the conventional turbulence generator (Cases 1a and 2a) and the fractal grid (Cases 3 and 4) are shown in Fig. 9 for the mean axial velocities and in Fig. 10 for the axial and radial fluctuations. A reduced mean axial velocity was observed for both turbulence generators when additional impact plates were used. However, no significant decrease in turbulence fluctuations were found when combining fractal based turbulence generation with impact plates. Accordingly, the fractal grids have the 
notable advantage of removing the sensitivity to upstream conditions while maintaining significantly increased levels of turbulence.

The flow field differences outlined above were further analysed by applying a POD technique within the region $r= \pm D / 2$ to decompose the instantaneous vector fields into their empirical eigenfields in order to explore the flow dynamics. The method is explained in detail by Holmes et al. [62] and was successfully applied amongst others by Adrian et al. [63], who showed the effectiveness of POD as a filtering tool using PIV measurements. The first eigenmode obtained with the traditional perforated plates without (Case 1a) and with (Case 2a) impact plates shows a rotational motion close to the stagnation point that causes the stagnation plane to tilt. The second mode is an axial movement causing a stagnation plane shift, as previously analysed by Geipel et al. [6]. The first five eigenmodes contain $60 \%$ of the total kinetic energy without impact plates and $39 \%$ with impact plates. The kinetic energy in the first mode is reduced from $31.2 \%$ to $17.6 \%$ and second mode from $15.5 \%$ to $11.1 \%$ with fitted impact plates. Hence, the increase in the fluctuations using traditional perforated plates is more likely to be caused by coherent flow structures generated by the four high velocity inlet jets impinging on the turbulence generator.

The fractal generated turbulence without impact plates (Case 3) contained $20.2 \%$ of the kinetic energy in the first mode and $16.8 \%$ in the second mode. The additional impact plates (Case 4) reduced the first mode to about $17.5 \%$ and the second to about $12.8 \%$. Furthermore, a mode switch, as compared to the conventional turbulence generators, was observed. The lower proportion of the kinetic energy contained in the first and second modes for the fractal grid turbulence generator suggests that the turbulence intensities of $\approx 20 \%$ of the bulk velocity at the nozzle exit plane are not 
dominated by the first two modes. The results for fractal grids are hence unlike traditional perforated plates at comparable levels of fluctuations and generate turbulence with a different structure by exciting a larger number of modes. The impact upon premixed turbulent flames is explored below.

\subsection{Combusting flows}

Velocity and scalar statistics are presented with a focus on the burner axis and the symmetry plane between the nozzles. The eight lean methane, propane and ethylene flames cover a range of stoichiometries $(0.70 \leq \phi \leq$ 1.0) and extend previous work of Lindstedt et al. [12] to cover increased turbulent intensities while maintaining the bulk strain rates. Results from additional vector field analyses and the flame front detection algorithm are also presented alongside conditional and unconditional velocity statistics,

with $\bar{Q}$ indicating a conventional and $\widetilde{Q}$ a Favre average for the property $Q$, and an evaluation of alternative methods for the determination of the turbulent burning velocity. The structure of the reacting turbulent flow field is further analysed using the POD and CPOD techniques outlined above.

\subsubsection{Analysis of the flow field}

The 2D detection algorithm developed by Geipel et al. [6] was used to determine the instantaneous stagnation point location and to quantify the flame movement caused by turbulence and any low frequency bulk flow instabilities. Three examples of stagnation point location statistics and the pdf for the overall tilt of the stagnation plane are given in Fig. 11 for premixed methane/air flames with $\phi=0.9$. The stagnation point essentially moves in an envelope of $4 \times 5 \mathrm{~mm}$ in the axial and radial directions. The results suggest that the turbulence intensity (cf. Fig. 10) impacts the width 
of the pdf with a substantial reduction observed for the perforated plates with a fitted impact plate. The fractal grid provides a narrower pdf than the perforated plate for similar turbulence levels. The axial movement is in good agreement with findings for the isothermal flow field [6], while the movement in the radial direction increased by up to $2 \mathrm{~mm}(\geq 50 \%)$ due to combustion. A slight bias towards a positive stagnation point tilt was also observed for all three flow configurations. The effect was investigated by comparing four different measurement planes resulting from successive $45^{\circ}$ rotations for the configuration using fractal grids with additional impact plates shown in Fig. 12. The stagnation point movement for all four positions was within a $3 \times 4 \mathrm{~mm}$ envelope, compared to the estimated integral length scale of $3.5 \mathrm{~mm}$ in the reactants, suggesting a modest increase in bias for the first measurement plane featuring a $45^{\circ}$ shift with respect to the largest fractal cross.

The increased radial movement of the instantaneous stagnation point was investigated further by an analysis of the first three eigenmodes for premixed methane flames with $\phi=0.9$ as shown in Fig. 13. It is apparent that combustion typically modifies or changes the dominant eigenmodes as compared to the isothermal flow. The first eigenmode for the case with conventional perforated discs without impact plates contains $20.6 \%$ of the total kinetic energy and remains a rotational motion close to the nominal stagnation point. The latter is axially skewed resulting in the broad pdf of the stagnation plane angle shown in Fig. 11. The same first mode was also found for the fractal grids with impact plates. However, the kinetic energy was lower at approximately $10 \%$ of the total. A direct consequence is a much narrower pdf of the rotation angle at comparable levels of fluctuations. In the presence of combustion, a new radial eigenmode was also observed as shown 
in Fig. 13. The associated kinetic energy was found to have a direct influence on the fluctuation levels in the vicinity of the stagnation plane and to lead to an increase of up to $100 \%$ as compared the corresponding isothermal flow as shown in Fig. 14. It has been shown by Goh et al. [22] that conditional POD (CPOD) is required to determine the energy distribution and the hybrid structures obtained using conventional POD are only presented to show the relative effects of different flow configurations.

Kostiuk et al. [11] applied different limiting values on the maximum axial movement of the stagnation point away from the nominal location in an effort to assess the potential impact of low frequency instabilities on velocity statistics and flow asymmetries. In the current work, the filtering algorithm of Geipel et al. [6] was used to investigate the impact of stagnation point movement on the velocity statistics. The current filter is based on the 2D detection algorithm discussed above and rejects all PIV derived vector fields where the stagnation point location falls outside a chosen circular domain of fixed radius. Filtered velocity statistics for methane/air flames using a threshold of $3.5 \mathrm{~mm}$, close to the integral length scale, show minimal impact on the axial mean and fluctuating velocity statistics and only a slight improvement in symmetry. The radial fluctuations were reduced by $\simeq 7.4 \%$ for $\phi=0.9$ and $\simeq 4.8 \%$ for $\phi=0.8$ at the nominal stagnation point. A further reduction in the radial fluctuations was found when the threshold was decreased to $2.5 \mathrm{~mm}$ though, as pointed out by Geipel et al. [6], it is probably not reasonable to apply a filter less than the integral length scale. Filtering at $3.5 \mathrm{~mm}$ leads to a rejection between $9.0 \%$ and $10.9 \%$ of the PIV images, whereas a threshold of $2.5 \mathrm{~mm}$ rejects up to $33.1 \%$. The resulting profiles are shown in Fig. 15 and the same trends were found for all fuels and stoichiometries. Given the modest impact of filtering on velocity statistics, 
subsequent graphs present unfiltered data and the error bars include both measurement uncertainties and flow asymmetries.

\subsubsection{Velocity statistics in fractal grid generated turbulence}

The discussion of velocity statistics for combusting flows is focussed on fractal grid generated turbulence with fitted impact plates (Case 4) with selected further comparisons to highlight differences compared to perforated plates. Isothermal results show that this configuration effectively removes low frequency flame movement and suppresses the impact of upstream conditions while producing velocity fluctuations $\simeq 20 \%\left(130 \leq R e_{t} \leq 318\right)$ of the corresponding bulk velocity.

Comparisons of normalised mean axial velocities along the burner centreline and corresponding radial velocities along the stagnation plane normal to the nozzles are shown in Fig. 16 for methane and propane flames at $U_{b}=4.0 \mathrm{~m} / \mathrm{s}$ and for ethylene flames at $U_{b}=8.0 \mathrm{~m} / \mathrm{s}$. The flames cover a range of stoichiometries. An acceleration in the axial direction, due to the reduction in density, can be seen for all fuels. The velocity increase is less noticeable for leaner flames due to the lower heat release. An acceleration in the radial direction from zero at the stagnation point up to $1.25-1.75 U_{b}$ at approximately half a nozzle diameter was found for all fuels. A decrease in the acceleration of the radial velocities was also evident at lower stoichiometries. A comparison of radial velocities with the corresponding isothermal flow [6] shows that the mean radial velocity is increased by a factor of two due to volume expansion caused by combustion.

Axial and radial fluctuations along the burner centreline are shown in Fig. 17 for methane and propane flames with $U_{b}=4.0 \mathrm{~m} / \mathrm{s}$ and for ethylene flames with $U_{b}=8.0 \mathrm{~m} / \mathrm{s}$. A comparison of the three fuels at a stoichiometry 
of 0.80 shows that the normalised axial fluctuations increase to a maximum of $0.25 U_{b}$ at the stagnation point for ethylene flames, whereas methane and propane flames showed slightly lower values of approximately $0.22 U_{b}$. All axial fluctuations increase ahead of the flame front and, as the flow accelerates in the axial direction, the turbulent intensities reduce before increasing again to reach their maximum at the stagnation point. The complex pattern of flame and flow induced turbulence generation/destruction is characteristic of opposed jet flames. Radial fluctuations stay constant up to a distance of $0.18 \mathrm{D}$ and increase to a maximum value of $0.30 U_{b}$ for methane and propane flames at $\phi=0.90$. Leaner flames at a stoichiometry of $\phi=0.80$ show a reduction of the radial fluctuations by $2.5 \%$. Values between $0.26-0.275 U_{b}$ were obtained for all three fuels at $\phi=0.80$, which suggests that the radial fluctuations are not dependent on the fuel type. The normalised fluctuations obtained for the isothermal flows at the nominal stagnation point were of the order of $0.28 U_{b}$ in the axial and $0.16 U_{b}$ in the radial directions (see Fig. 14), respectively. The corresponding values for the combusting flow are $0.185-$ $0.27 U_{b}$ in the axial and $0.24-0.30 U_{b}$ radial directions for equivalence ratios from 0.70 to 0.90 . The results suggest that for the current conditions, the axial stresses are comparable to the isothermal flow whereas radial stresses increase significantly due to combustion.

Profiles of measured axial and radial fluctuations along the stagnation plane showed a good degree of symmetry with respect to the stagnation point as shown in Fig. 18. Fluctuation levels increased in the radial direction in the presence of combustion. By contrast, isothermal flows [6] only showed an increase in the axial Reynolds stress component from $0.20 U_{b}$ at the stagnation point to $0.29 U_{b}$ at a radial distance of approximately $0.5 \mathrm{D}$, while the radial component decreased slightly from $0.12 U_{b}$ to $0.10 U_{b}$. The 
increase of the radial stress component for combusting flows is consistent with the increased radial movement illustrated by the broader probability density function for the stagnation point location shown in Fig. 11.

Goh et al. [22] outlined the extension of the POD technique to combusting flows by applying the density segregation technique to provide conditional POD data. Results for the current cases are shown in Figs. 19 and 20. As shown in Fig. 19, the introduction of impact plates in combination with conventional turbulence generating plates leads to a substantial decrease in turbulence intensities. Hence, such a combination is not feasible when elevated turbulence levels are sought. By contrast, the introduction of impact plates has only a modest impact on the generated turbulence levels when fractal grids are used. The implication is that the influence of the flow conditions upstream of the fractal grid has a much reduced impact. It is further readily apparent that the unconditional POD results are biased towards the reactants and that the presence of the flame reduces the levels of turbulence in the reactants as compared to the isothermal flow. Results obtained for six methane and propane flames are shown in Fig. 20 for fractal grids combined with impact plates and the trends are consistent with the methane case discussed above.

\subsubsection{Reaction progress variable statistics}

The first two moments of the reaction progress variable statistics derived from PIV-images obtained using aluminium oxide seeding in combination with the flame front detection algorithm are shown in Fig. 21. The methane and propane flames featured $U_{b}=4.0 \mathrm{~m} / \mathrm{s}$ and ethylene flames $U_{b}=8.0 \mathrm{~m} / \mathrm{s}$ with equivalence ratios between 0.70 and 0.90 . The average flame front location is evident from the mean reaction progress variable. It can be seen 
that the flames move closer together when approaching the lean extinction limits. Leaner fuel/air mixtures, e.g. methane or propane at $\phi=0.80$ or ethylene at $\phi=0.70$ show a reduction of the mean progress variable at the nominal stagnation point. Accordingly, reactants can also be found along the stagnation plane. In general, propane flames appear more wrinkled and curved towards their reactant streams while methane flames are flatter, thinner and with the flame brushes closer together (cf. Luff et al. [17] and Yoshida et al. [67]). Variations in the separation distance, also found by Sato [68] and Ishizuka and Law [69] for laminar flames, is likely due to differences in thermochemistry and transport properties with the impact of the Lewis number discussed below.

A comparison of methane and propane flames shows that for the same bulk strain rate, the twin methane flames were on average closer together with a higher probability of finding reactants at the nominal stagnation point. It should, however, also be noted that the propane flames extinguished at an equivalence ratio of 0.79 whereas methane flames were stable even below 0.75. At equal stoichiometries, propane has a higher laminar burning velocity and a higher adiabatic flame temperature. The higher Lewis number (see Table 1) may be the reason for the earlier extinction of the propane flames. Ethylene flames withstood bulk velocities in excess of $8.0 \mathrm{~m} / \mathrm{s}$ with progress variable statistics showing similar trends to the other fuels when subjected to a much larger total strain as also shown in Fig. 21. The overall behaviour of the fuels suggests that both chemical rate and transport processes affect progress variable statistics at turbulent Reynolds numbers $>300$. 


\subsubsection{Conditional velocities and scalar fluxes}

The reaction progress variable information obtained from the flame front detection algorithm was used to generate conditional statistics following a procedure similar to that by Kalt et al. [70]. Hence, the mixture was treated as reactants if the instantaneous average value of the reaction progress variable in an aligned interrogation window was less than 50\% and otherwise as products. The associated spatial distribution of velocity vectors in combination with the progress variable profiles made it possible to obtain conditional velocities and scalar fluxes. The corresponding laminar flame properties, such as reactant $\left(\rho_{r}\right)$ and product $\left(\rho_{p}\right)$ densities and Lewis numbers for the deficient reactant were computed using a subset of a detailed chemical kinetic mechanism [45] and are presented in Table 1.

Statistics were calculated along the stagnation point streamline for the conditional axial velocities of reactants $\left(\bar{u}_{r}\right)$ and products $\left(\bar{u}_{p}\right)$, as well as the scalar flux $\left(\overline{u^{\prime} c^{\prime}}\right)$. The data was analysed in the context of the BrayMoss-Libby formalism [71] with the mean slip velocity $\left(u_{s}\right)$, defined as the difference between the mean product and reactant velocities [72], also calculated. Results for $\bar{u}_{r}, \bar{u}_{p}$ and $u_{s}$ obtained for $\phi=0.80$ and 0.90 are presented in Fig. 22 for methane flames and in Fig. 23 for propane flames. The quantities are plotted against the corresponding mean reaction progress variable. The slip velocity is almost constant in the region $0.2 \leq \bar{c} \leq 0.7$ for all cases. Towards the leading edge of the flames the reactants decelerate leading to a rapid decrease in the slip velocities. A deceleration of both reactant and product velocities is apparent further towards the product side. The results suggest that the flow along the centreline can be classified in three regions. Two of the regions $(\bar{c} \leq 0.2$ and $\bar{c} \geq 0.7)$ show deceleration and an increase 
in pressure while in a narrow region in physical space, corresponding to roughly $3 \mathrm{~mm}(0.2 \leq \bar{c} \leq 0.7)$, the dominant effect is a flow acceleration caused by the heat release across the flame brush.

The turbulent scalar fluxes along the stagnation point streamline are presented as a function of the reaction progress variable in Fig. 24 and axial distance in Fig. 25. The latter figure also shows the estimated total uncertainties and it is apparent that the scatter increases significantly for the stoichiometric flames and hence the corresponding conditional velocity analysis is subject to increased uncertainties. However, it is apparent that the flames are increasingly in the transition region between gradient (GD) and counter-gradient (CGD) diffusion as the equivalence ratio is increased and the experimental scatter may be influenced by the delicate balance of the turbulent transport mechanisms. The increased scatter is also partly due to more prevalent preheating $(\leq 50 \mathrm{~K})$ of the reactant streams, particularly from the upper nozzle, as a result of the increased rate of heat release. The impact of the latter is also evident for the ethylene flames. However, the change in the sign of the scalar flux can readily be analysed using BML theory [71] and is accordingly determined by the slip velocity as shown in Eq. (6).

$$
\widetilde{u^{\prime \prime} c^{\prime \prime}}=\widetilde{c}(1-\widetilde{c})\left(\bar{u}_{p}-\bar{u}_{r}\right)=\widetilde{c}(1-\widetilde{c}) u_{s}
$$

A negative scalar flux suggests gradient diffusion and a positive value counter gradient diffusion and the current experimental resolution is sufficient to show the narrow region of GD at the leading edge (cf. Fig. 24). Veynante et al. [73] derived the Bray number as a criterion for the transition between 
GD and CGD in freely propagating flames with subsequent refinements provided [74, 75]. Chen and Bilger [76] further analysed the transition from GD to CGD using Bunsen flames. Lindstedt and Váos [3] derived an alternative relationship based on the balance between mean strain (production term tensor $P_{i j}$ ) and mean pressure gradient effects (tensor $\Phi_{i j}$ ). However, in the turbulent opposed jet configuration the geometry imposed pressure gradient exerts a significant influence on the transition from GD to CGD. Efforts have been made to account for such effects [70, 74], though a comprehensive evaluation of the resulting model formulations present difficulties in the current context. However, the current data, see Fig. 25, shows a gradual transition between GD and CGD regimes as $\phi$ is increased. The result is consistent with increases in the heat release gradually overcoming the adverse effect of the pressure gradient leading to an expanding local region of CGD with increasingly positive fluxes.

\subsubsection{Turbulent burning velocity determinations}

Multiple definitions of the turbulent burning velocity (e.g. Bray [72]) have been advanced and Driscoll [77] showed that definitions (e.g. [78, 79]) are not equivalent. The matter is explored quantitatively below. Bray [72] derived the turbulent burning velocity $\left(s_{T, 1}\right)$ as twice the magnitude of the slip velocity at the leading edge of the flame. Due to the steep gradients in slip velocities close to the leading edge the direct evaluation can result in significant uncertainties while the determination of the relevant root to Eq. (7) using linear interpolation remains amenable.

$$
s_{T, 1}=-2\left(u_{s}\right)_{\widetilde{c} \rightarrow 0},
$$




$$
\therefore \text { solve } u_{s} \quad: \quad \rho_{r} \bar{u}_{r}+2 \bar{\rho} u_{s}=0
$$

Lawn and Schefer [79] provided a simple definition of the turbulent burning velocity $\left(s_{T, 2}\right)$ based on the mean flow velocity at the leading edge $(\bar{c}=0.02)$ as shown in Eq. (8). From a theoretical point of view, Catlin and Lindstedt [80] have shown that the burning velocity eigenvalue $(\Lambda)$ is strongly dependent upon the $c$ iso-contour $\left(c^{*}\right)$ used for the determination with $\Lambda=0.54$ for $\bar{c}^{*}=10^{-1}, \Lambda=1.25$ for $\bar{c}^{*}=10^{-2}, \Lambda=1.63$ for $\bar{c}^{*}=10^{-3}$ and $\Lambda=1.80$ for $\bar{c}^{*}=10^{-4}$ and the method can be expected to have a tendency to produce lower values.

$$
s_{T, 2}=\left.\bar{u}\right|_{\bar{c}^{*}=0.02}
$$

Driscoll [77] proposed the use of the local minimum in the mean velocity profile as an indication of the leading edge location. However, the axial mean velocity profiles shown in Fig. 16 suggest that local minima are only prominent at higher equivalence ratios. It is accordingly proposed here that the local minimum in the gradient of the axial velocity profile should used to indicate the location of the leading edge of the flame and hence used to determine the burning velocity $\left(s_{T, 3}\right)$ as shown in Eq. (9). The velocity gradients were determined by backward differencing with quadratic least square fits used for noise reduction.

$$
s_{T, 3}=\left.\bar{u}\right|_{\frac{d \bar{u}}{d x} \rightarrow 0}
$$

The centreline profiles of $\bar{\rho}, \bar{c}, \bar{u}, \bar{u}_{r}, u_{s}$ and $d \bar{u} / d x$ were used to determine turbulent burning velocities through the alternative definitions $\left(s_{T, 1}\right.$, 
$s_{T, 2}$ and $\left.s_{T, 3}\right)$ and statistics from the lower nozzle. The results are presented in Table 2 and values agree to within $20 \%$ - comparatively close given the relatively wide spread highlighted in the reviews by Driscoll [77] and Lipatnikov and Chomiak [81]. Overall, the values of $s_{T, 3}$ are larger than the corresponding $s_{T, 2}$ determination (based on $\bar{c}^{*}=0.02$ ) as the point of evaluation was consistently closer to the true leading edge with mean velocities corresponding larger. However, it can be seen that there are exceptions with values of $s_{T, 1}$ lower than $s_{T, 2}$ at an equivalence ratio of 0.80 , which may reflect the evaluation difficulties associated with the leading edge slip velocity as discussed above. For comparison purposes [22], a theoretical estimate can readily be provided via a KPP analysis $[82,83]$ applied to a fractal flame surface area based rate expression $[3,84]$. The resulting scaling $\left(\Lambda_{K P P}=2\right)$ is given in Eq. (10) with the required parameters listed in Table 1.

$$
s_{T, K P P}=\Lambda_{K P P} \sqrt{C_{R}(3 / 2)\left(C_{\mu} / \sigma_{\mathrm{Sc}}^{t}\right)\left(s_{\mathrm{L}} / v_{\kappa}\right)} \quad u^{\prime} \quad v_{\kappa}=\left(\nu_{r} \epsilon_{r}\right)^{1 / 4}
$$

In the theoretical estimation of the turbulent burning velocity $\left(s_{T}=s_{L}+\right.$ $\left.s_{T, K P P}\right)$, the velocity fluctuations $\left(u^{\prime}\right)$ normal to the flame propagation direction were used. The values for $s_{T} / u^{\prime}$ shown in Table 2 provide reasonable agreement with a tendency to underpredict the experimental values. The latter is particularly noticeable for ethylene. Fractal grids have been found to substantially enhance turbulent diffusivities [26] due to the multi-scale nature of the generated turbulence and a modest reduction in $\sigma_{S c}^{t}$ would improve the overall agreement.

\section{Conclusions}

The approach to extinction of fuel lean turbulent premixed flames in fractal grid generated multi-scale turbulence was studied for methane, propane 
and ethylene flames using PIV with the flows to both nozzles seeded with $1 \mu \mathrm{m}$ silicon oil droplets or $3 \mu \mathrm{m} \mathrm{Al}_{2} \mathrm{O}_{3}$ particles. The obtained data include velocity and reaction progress variable statistics as well as conditional velocities and scalar fluxes. The fractal grids were used to ameliorate limitations associated with conventional perforated plate generators with the turbulent Reynolds number range moved from 50-120 to 130-318. Furthermore, optimal grid configurations were determined with particular emphasis on reducing the impact of the flow upstream of the turbulence generators in order to facilitate simpler boundary conditions for computational studies. It has been shown that the current fractal grids have the notable advantage of removing the sensitivity to upstream conditions while maintaining significantly increased levels of turbulence.

Dilatation due to the heat release across the flame front induces a positive velocity gradient which leads to an acceleration of the flow. The reduction in pressure gradient due to dilatation results in a drop in the axial fluctuations along the axis for all measured fuel types. Leaner flames showed less acceleration across the flame front due to lower heat release. The same trends were found for the mean radial velocity and the fluctuations along the stagnation plane. Radial strain rates along the stagnation plane reached their maximum value at a radial distance of $0.3 \mathrm{D}$ for all fuel types. The radial strain rates measured in the reacting flow show an increase $\simeq 100 \%$, compared to isothermal measurements [6], that is directly related to the higher radial velocity gradients caused by heat release along the stagnation plane.

The resulting flow structures were further analysed using POD and CPOD with results confirming that conventional (unconditional) POD is biased towards the reactants. The use of CPOD enables a more detailed analysis of the flow field with the impact of the flame on both reactant and 
product fields readily segregated. The technique shows that conventional POD leads to a hybrid biased towards the reactants. Probability density functions were determined for the instantaneous location of the stagnation point to eliminate the possibility of low frequency bulk motion distorting velocity statistics. The PDFs of flame curvature were determined using a multi-step flame front detection algorithm with estimates of the turbulent burning velocity provided along with a discussion of alternative determination methods. The data sets show that fractal grids generate multi-scale broadband turbulence and present an opportunity for a systematic evaluation of calculation methods for premixed turbulent flames that undergo a transition from non-gradient to gradient turbulent transport while approaching extinction. It is further shown that reasonable agreement with models for the turbulent burning velocity is obtained for the current set of flames. The lean extinction limits for methane and propane flames suggest an influence of the Lewis number and were, respectively, at $\phi=0.73$ and $\phi=0.79$ for a fixed bulk velocity of $4.0 \mathrm{~m} / \mathrm{s}$ with ethylene flames extinguishing at $\phi=0.66$ at $8.0 \mathrm{~m} / \mathrm{s}$. It is expected that the current data sets will be of particular value in the development of generalised models that account for the transition between gradient and non-gradient transport in the presence of imposed pressure gradients.

\section{Acknowledgements}

The research was carried out with the financial support of the Office of Naval Research Global under grant N62909-12-1-7127 and European Office of Aerospace Research and Development/Air Force Office of Scientific Research under grant FA8655-13-1-3024. The support of Dr Gabriel Roy, Dr 
Chiping Li and Dr Gregg Abate is gratefully acknowledged.

\section{References}

[1] K.N.C. Bray, M. Champion, P.A. Libby, Premixed flames in stagnating turbulence Part III - The $\widetilde{k}-\widetilde{\epsilon}$ theory for reactants impinging on a wall, Combust. Flame 91 (1992) 165-186.

[2] R.P. Lindstedt, E.M. Váos, Second moment modeling of premixed turbulent flames stabilized in impinging jet geometries, Proc. Combust. Inst. 27 (1998) 957-962.

[3] R.P. Lindstedt, E.M. Váos, Modeling of premixed turbulent flames with second moment methods, Combust. Flame 116 (1999) 461-485.

[4] D. Geyer, A. Kempf, A. Dreizler, J. Janicka, Scalar dissipation rates in isothermal and reactive turbulent opposed-jets: 1-D-Raman/Rayleigh experiments supported by LES, Proc. Combust. Inst. 30 (2005) 681689.

[5] D. Geyer, A. Kempf, A. Dreizler, J. Janicka, Turbulent opposed-jet flames: A critical benchmark experiment for combustion LES, Combust. Flame 143 (2005) 524-548.

[6] P. Geipel, K.H.H. Goh, R.P. Lindstedt, Fractal-generated turbulence in opposed jet flows, Flow, Turbul. Combust. 85 (2010) 397-419.

[7] L.W. Kostiuk, K.N.C. Bray, R.K. Cheng, Experimental study of premixed turbulent combustion in opposed streams. Part I - Nonreacting flow field, Combust. Flame 92 (1993) 377-395. 
[8] E. Korusoy, J.H. Whitelaw, Opposed jets with small separations and their implications for the extinction of opposed flames, Exp. Fluids 31 (2001) 111-117.

[9] R.P. Lindstedt, D. Luff, J.H. Whitelaw, Velocity and strain-rate characteristics of opposed isothermal flows, Flow, Turbul. Combust. 74 (2005) 169-194.

[10] C. Mounaïm-Rousselle, I. Gökalp, Turbulent premixed combustion in counterflow geometry. the influence of a coflow, in: Spring Annual Meeting of the Western States Section of the Combustion Institute, 1993.

[11] L.W. Kostiuk, I. Shepherd, K.N.C. Bray, Experimental study of premixed turbulent combustion in opposed streams. Part III - Spatial structure of flames, Combust. Flame 118 (1999) 129-139.

[12] R.P. Lindstedt, D. Luff, J.H. Whitelaw, Velocity fields of lean premixed turbulent opposed jet flames, Proc. Combust. Inst. 31 (2007) 14591466.

[13] L.W. Kostiuk, K.N.C. Bray, R.K. Cheng, Experimental study of premixed turbulent combustion in opposed streams. Part II - Reacting Flow Field and Extinction, Combust. Flame 92 (1993) 396-409.

[14] C. Mounaïm-Rousselle, I. Gökalp, Strain effects on the structure of counterflowing turbulent premixed flames, Proc. Combust. Inst. (1994) $1199-1205$.

[15] K. Sardi, J.H. Whitelaw, Extinction timescales of periodically strained, lean counterflow flames, Exp. Fluids 27 (1999) 199-209. 
[16] E. Korusoy, J.H. Whitelaw, Extinction and relight in opposed flames, Exp. Fluids 33 (2002) 75-89.

[17] D. Luff, E. Korusoy, R.P. Lindstedt, J.H. Whitelaw, Counterflow flames of air and methane, propane and ethylene, with and without periodic forcing, Exp. Fluids 35 (2003) 618-626.

[18] L.W. Kostiuk, Premixed turbulent combustion in counterflowing streams, Ph.D. thesis, Churchill College, University of Cambridge (1991).

[19] G. Coppola, A. Gomez, Experimental study of highly turbulent isothermal opposed-jet flows, Phys. Fluids 22 (2010) 105-101.

[20] G. Coppola, B. Coriton, A. Gomez, Highly turbulent counterflow flames: A laboratory scale benchmark for practical systems, Combust. Flame 156 (2009) 1834-1843.

[21] A. Gomez, B. Coriton, J.H. Frank, Highly turbulent counterflow flames: A laboratory-scale benchmark for turbulent combustion studies, Eleventh International Workshop on Measurement and Computation of Turbulent Flames (TNF11), Darmstadt, July 26 - 28, 2012

[22] K. H. H. Goh, P. Geipel, F. Hampp, R. P. Lindstedt, Flames in fractal grid generated turbulence, Fluid Dyn. Res. 45 (6) (2013) 061403.

[23] J.C. Vassilicos, J.C.R. Hunt, Fractal dimensions and spectra of interfaces with application to turbulence, Proc. Math. Phys. Sci. 435 (1895) (1991) 505-534.

[24] R.E. Seoud, J.C. Vassilicos, Dissipation and decay of fractal generated turbulence, Phys. Fluids 19, 105108 (2007) 1-11. 
[25] D. Hurst, J.C. Vassilicos, Scaling and decay of fractal-generated turbulence, Phys. Fluids 19, 035103 (2007) 1-31.

[26] S. Laizet, J.C. Vassilicos, Fractal space-scale unfolding mechanism for energy-efficient turbulent mixing, Phys. Review E 86046302 (2012).

[27] Y. Sakai, K. Nagata, H. Suzuki, K. Hoshino, O. Terashima, Y.Ito, Development of scalar mixing layer in regular/fractal grid turbulence, 2nd UK-Japan bilateral and 1st ERCOFTAC Workshop on Turbulent flows generated/designed in multiscale/fractal ways, London 26-27 March 2012 .

[28] K.H.H. Goh, P. Geipel, F. Hampp, R.P. Lindstedt, Regime transition from premixed to flameless oxidation in turbulent JP-10 flames, Proc. Combust. Inst. 34 (2013) 3311 - 3318.

[29] M. de Joannon, A. Matarazzo, P. Sabia, A. Cavaliere, MILD combustion in homogeneous charge diffusion ignition (HCDI) regime, Proc. Combust. Inst. 31 (2007) 3409-3416.

[30] W.K. George, H.J. Hussein, Locally axisymmetric turbulence J. Fluid Mech. 233(1) (1991) 1-23.

[31] D. Geyer, S. Omar, A. Nauert, A. Ludwig, A. Dreizler, J. Janicka, A comprehensive characterisation of a turbulent opposed jet flame by 1D-Raman/Rayleigh, 2D-LIF and 2D-LDV, VDI Berichte 1750 (2003) $435-440$.

[32] E. Mastorakos, A.M.K.P. Taylor, J.H. Whitelaw, Extinction and temperature characteristics of turbulent counterflow diffusion flames with partial premixing, Combust. Flame 91 (1992) 40-54. 
[33] E. Mastorakos, A.M.K.P. Taylor, J.H. Whitelaw, Scalar dissipation rate at the extinction of turbulent counterflow nonpremixed flames, Combust. Flame 91 (1992) 55-64.

[34] E. Mastorakos, A.M.K.P. Taylor, J.H. Whitelaw, Extinction of turbulent counterflow flames with reactants diluted by hot products, Combust. Flame 102 (1995) 101-114.

[35] K. Sardi, A.M.K.P. Taylor, J.H. Whitelaw, Conditional scalar dissipation statistics in a turbulent counterflow, J. Fluid Mech. 361 (1998) $1-24$.

[36] K. Sardi, A.M.K.P. Taylor, J.H. Whitelaw, Extinction of turbulent counterflow flames under periodic strain, Combust. Flame 120 (2000) $265-284$.

[37] K.H.H. Goh, Investigation of conditional statistics in premixed combustion and the transition to flameless oxidation in turbulent opposed jets, PhD Thesis, Imperial College, October 2013.

[38] A. Høst-Madsen, A. H. Nielsen, Accuracy of PIV measurements in turbulent flows, Proc. ASME/JSME Fluid Eng. Conf., ASME 229 (1995) $481-488$.

[39] K.P. Angele, B. Muhammad-Klingmann, A simple model for the effect of peak-locking on the accuracy of boundary layer turbulence statistics in digital PIV, Exp. Fluids 38 (2005) 314-347.

[40] J. Westerweel, Fundamentals of digital particle image velocimetry, Meas. Sci. Technol. 8 (1997) 1379-1392. 
[41] M. Raffel, C. Willert, J. Kompenhans, Particle Image Velocimetry, Springer Verlag, Berlin, Germany, 1998.

[42] A.K. Prasad, R.J. Adrian, C.C. Landreth, P.W. Offutt, Effect of resolution on the speed and accuracy of particle image velocimetry interrogation, Exp. Fluids 13 (1992) 105-116.

[43] E.A. Cowen, S.G. Monismith, A hybrid digital particle tracking velocimetry technique, Exp. Fluids 22 (1997) 199-211.

[44] D. Han, M. Mungal, Simultaneous velocity and CH distributions. Part I: jet flames in a co-flow, Combust. Flame 132 (2003) 565-590.

[45] R.K. Robinson, R.P. Lindstedt, On the chemical kinetics of cyclopentadiene oxidation, Combust. Flame 158 (2011) 666-686.

[46] C.J. Sung, J.S. Kistler, M. Nishioka, C.K. Law, Further studies on effects of thermophoresis on seeding particles in LDV measurements of strained flames, Combust. Flame 105 (1996) 189-201.

[47] C.J. Sung, C.K Law, R.J. Axelbaum, Thermophoretic effects on seeding particles in LDV measurements on flames, Combust. Sci. Technol. 99 (1994) 119-132.

[48] A. Gomez, D.E. Rosner, Thermophoretic effects on particles in counterflow laminar diffusion flames, Combust. Sci. Technol. 89 (1993) 335-362.

[49] G. Amantini, J.H. Frank, B.A.V. Bennett, M.D. Smooke, A. Gomez, Comprehensive study of the evolution of an annular edge flame during extinction and reignition of a counterflow diffusion flame perturbed by vortices., Combust. Flame 150 (2007) 292-319. 
[50] H.K. Chelliah, C.K. Law, T. Ueda, M.D. Smooke, F.A. Williams, An experimental and theoretical investigation of the dilution, pressure and flow-field effects on the extinction condition of methane-air-nitrogen diffusion flames, Proc. Combust. Inst. 23 (1991) 503-511.

[51] S.A. Kaiser, J.H. Frank, M.B. Long, Use of Rayleigh imaging and ray tracing to correct for beam-steering effects in turbulent flames, Appl. Optics 44 (2005) 6557-6564.

[52] J.H. Frank, S.A. Kaiser, M.B. Long, Multiscalar imaging in partially premixed jet flames with argon dilution, Combust. Flame 143 (2005) $507-523$.

[53] B. Böhm, Experiments for the validation of Large Eddy Simulations: Turbulence-flame interactions in extinguishing turbulent opposed jet flames, Ph.D. thesis, TU Darmstadt, Germany (2008).

[54] A. Steinberg, J.F. Driscoll, S. Ceccio, Measurements of turbulent premixed flame dynamics using cinema stereoscopic PIV, Exp. Fluids 44 (6) (2008) 985-999.

[55] S. Pfadler, F. Beyrau, A. Leipertz, Flame front detection and characterization using conditioned particle image velocimetry (CPIV), Optics Express 15 (23) (2007) 15444-15456.

[56] J. Kerl, T. Sponfeldner, F. Beyrau, An external Raman laser for combustion diagnostics, Combust. Flame 158 (2011) 1905-1907.

[57] I. Shepherd, R. Cheng, P. Goix, The spatial scalar structure of premixed turbulent stagnation point flames Proc. Combust. Inst. 23 (1) (1991) $781-787$. 
[58] C. Tomasi, R. Manduchi, Bilateral filtering for gray and color images, IEEE International Conference on Computer Vision (1998) 839-846.

[59] S. Pfadler, M. Löffler, F. Dinkelacker, A. Leipertz, Measurement of the conditioned turbulence and temperature field of a premixed bunsen burner by planar laser Rayleigh scattering and stereo particle image velocimetry, Exp. Fluids 39 375-384.

[60] J. Canny, A computational approach to edge detection, IEEE Transactions on Pattern Analysis and Machine Intelligence 8 (6) (1986) 679 -698 .

[61] A. Datta, S. Parui, A robust parallel thinning algorithm for binary images, Pattern Recognition 27 (9) (1994) 1181-1192.

[62] P. Holmes, J. Lumley, G. Berkooz, Turbulence, coherent structures, dynamical systems and symmetry, Cambridge University Press, 1998.

[63] R.J. Adrian, K.T. Christensen, Z.-C Liu, Analysis and interpretation of instantaneous turbulent vector fields, Exp. Fluids 29 (2000) 275-290.

[64] N.W.H. Armstrong, K.N.C. Bray, Premixed turbulent combustion flowfield measurements using PIV and LST and their application to flamelet modelling of engine combustion, SAE Trans. 101(3) (1992) 2000-2011.

[65] E.J. Stevens, K.N.C. Bray, B. Lecordier, Velocity and scalar statistics for premixed turbulent stagnation flames using PIV, Proc. Combust. Inst. 27 (1) (1998) $949-955$.

[66] Y.C. Chen, Measurements of flame-front curvature based on Fourier transformation, Combust. Theory Model. 11 (3) (2007) 333-349. 
[67] A. Yoshida, T. Igarashi, Y. Kotani, Extinction of turbulent diffusion flames by Kolmogorov microscale turbulence, Combust. Flame 109 (1997) 669-687.

[68] J. Sato, Effect of Lewis number on extinction behaviour of premixed flames in a stagnating flow, Proc. Combust. Inst. 9 (1982) 1541-1548.

[69] S. Ishizuka, C.K. Law, An experimental study on extinction and stability of stretched premixed flames, Proc. Combust. Inst. 9 (1982) 327-335.

[70] P. Kalt, Y. Chen, R.W. Bilger, Experimental investigation of turbulent scalar flux in premixed stagnation-type flames, Combust. Flame 129 (4) (2002) 401-415.

[71] K.N.C. Bray, P.A. Libby, J.B. Moss, Unified modelling approach for premixed turbulent combustion Part 1 - General formulation, Combust. Flame 61 (1985) 87-102.

[72] K.N.C. Bray, Studies of the turbulent burning velocity, Proc. Roy. Soc. A431 (1990) 315-335.

[73] D. Veynante, A. Trouvé, K.N.C. Bray, T. Mantel, Gradient and counter-gradient scalar transport in turbulent premixed flames, J. Fluid Mech. 332 (1997) 263-293.

[74] D. Veynante, T. Poinsot, Effects of pressure gradients on turbulent premixed flames, J. Fluid Mech. 353 (1997) 83-114.

[75] P. Kalt, R.W. Bilger, Experimental characterisation of the $\alpha$-parameter in turbulent scalar flux for premixed combustion, Combust. Sci. Technol. 153 (1) (2000) $213-221$. 
[76] Y.-C. Chen, R.W. Bilger, Experimental investigation of threedimensional flame-front structure in premixed turbulent combustion I: Hydrocarbon/air Bunsen flames Combust. Flame 131 (2002) 400 435.

[77] J.F. Driscoll, Turbulent premixed combustion: Flamelet structure and its effect on turbulent burning velocities, Prog. Energy Combust. Sci. 34 (1) (2008) $91-134$.

[78] I.G. Shepherd, R.K. Cheng, The burning rate of premixed flames in moderate and intense turbulence, Combust. Flame 127 (3) (2001) 2066 $-2075$.

[79] C.J. Lawn, R. Schefer, Scaling of premixed turbulent flames in the corrugated regime, Combust. Flame 146 (1-2) (2006) 180 - 199.

[80] C.A. Catlin, R.P. Lindstedt, Premixed turbulent burning velocities derived from mixing controlled reaction models with cold front quenching, Combust. Flame 85 (1991) 427-439.

[81] A.N. Lipatnikov, J. Chomiak, Effects of premixed flames on turbulence and turbulent scalar transport, Prog. Energy Combust. Sci. 36 (2010) $1-102$.

[82] A. N. Kolmogorov, I. G. Petrovskii, N. S. Piskunov, A study of diffusion equation with a source term for application to a biologic problem, Bull. Moscow State Univ. Ser. A1 (1937) 1.

[83] B. Hakberg, A.D. Gosman, Analytical determination of turbulent flame speed from combustion models, Proc. Combust. Inst. 20 (1984) 225-232. 
[84] R.P. Lindstedt, V. Sakthitharan, in '8th Symp. Turbul. Shear Flows' pp. 22-5-1 - 22-5-6 (1991).

[85] N. K. Aluri, P. K. G. Pantangi, S. P. R. Muppala, F. Dinkelacker, A numerical study promoting algebraic models for the Lewis number effect in atmospheric turbulent premixed bunsen flames, Flow Turbul. Combust. 75 (1) (2005) 149-172. 


\section{List of Tables}

Table 1: Values of density used for calculations of the Favre averaged terms. Reactant $\left(\rho_{r}\right)$ and product $\left(\rho_{p}\right)$ densities. The Lewis number was defined for the deficient reactant (fuel) as $\sigma_{L e_{D}}=\sigma_{S c_{f u e l}} / \sigma_{P r}$ and laminar burning velocities obtained from calculations using detailed chemistry [45]. Rates of dissipation in the reactant streams $\left(\epsilon_{r}\right)$ were obtained using the conditional dissipation method defined by Goh et al. [22] as derived from the work by George and Hussein [30].

\begin{tabular}{|c|c|c|c|c|c|c|c|}
\hline Fuel & $\phi$ & $\begin{array}{c}\rho_{r} \\
{\left[\mathrm{kgm}^{-3}\right]}\end{array}$ & $\begin{array}{c}\rho_{p} \\
{\left[\mathrm{kgm}^{-3}\right]}\end{array}$ & $\begin{array}{c}\nu_{r} \times 10^{-5} \\
{\left[\mathrm{~m}^{2} \mathrm{~s}^{-1}\right]}\end{array}$ & $\begin{array}{c}\epsilon_{r} \\
{\left[\mathrm{~m}^{2} \mathrm{~s}^{-3}\right]}\end{array}$ & $\begin{array}{c}\mathrm{s}_{L} \\
{\left[\mathrm{~ms}^{-1}\right]}\end{array}$ & $\begin{array}{c}\sigma_{L e_{D}} \\
{[-]}\end{array}$ \\
\hline $\mathrm{CH}_{4}$ & 0.80 & 1.134 & 0.177 & 1.60 & 49.9 & 0.262 & 0.96 \\
$\mathrm{CH}_{4}$ & 0.90 & 1.129 & 0.168 & 1.59 & 53.0 & 0.325 & 0.96 \\
$\mathrm{CH}_{4}$ & 1.0 & 1.124 & 0.162 & 1.59 & 44.9 & 0.368 & 0.96 \\
\hline $\mathrm{C}_{3} \mathrm{H}_{8}$ & 0.80 & 1.192 & 0.176 & 1.50 & 48.9 & 0.293 & 1.82 \\
$\mathrm{C}_{3} \mathrm{H}_{8}$ & 0.90 & 1.193 & 0.168 & 1.49 & 45.7 & 0.362 & 1.82 \\
$\mathrm{C}_{3} \mathrm{H}_{8}$ & 1.0 & 1.194 & 0.164 & 1.48 & 42.5 & 0.412 & 1.80 \\
\hline $\mathrm{C}_{2} \mathrm{H}_{4}$ & 0.70 & 1.178 & 0.179 & 1.53 & 197 & 0.309 & 1.32 \\
$\mathrm{C}_{2} \mathrm{H}_{4}$ & 0.80 & 1.178 & 0.164 & 1.52 & 210 & 0.420 & 1.31 \\
\hline
\end{tabular}


Table 2: Values of different turbulent burning velocities and mean axial rms fluctuations $\left(u^{\prime}\right)$ evaluated as averages of upper and lower nozzles. Values of $s_{T, 1}, s_{T, 2}$ and $s_{T, 3}$ are derived from Eqs. (7), (8) and (9). Uncertainties in $u^{\prime}$ were evaluated as unbiased standard deviation from the mean of corresponding localised values for $s_{T, 1}, s_{T, 2}$ and $s_{T, 3}$. The theoretical values for $s_{T} / u^{\prime}=\left(s_{L}+s_{T, K P P}\right) / u^{\prime}$ were derived using Eq. (10) with values for $s_{L}$ and $\epsilon_{r}$ obtained from Table 1 with the reactant value of $v_{\kappa}=\left(\nu_{r} \epsilon_{r}\right)^{1 / 4}$. The value of $C_{R}$ was obtained as $4.0 / \sigma_{L e_{D}}$ [85] with resulting values for $s_{T} / u^{\prime}$ given below.

\begin{tabular}{|c|c|c|c|c|c|c|}
\hline Fuel & $\phi$ & $u^{\prime}[\mathrm{m} / \mathrm{s}]$ & $s_{T} / u^{\prime}[-]$ & $s_{T, 1} / u^{\prime}[-]$ & $s_{T, 2} / u^{\prime}[-]$ & $s_{T, 3} / u^{\prime}[-]$ \\
\hline $\mathrm{CH}_{4}$ & 0.80 & $0.90 \pm 0.03$ & 2.45 & $2.32 \pm 0.17$ & $2.36 \pm 0.16$ & $2.72 \pm 0.09$ \\
$\mathrm{CH}_{4}$ & 0.90 & $0.92 \pm 0.01$ & 2.75 & $2.87 \pm 0.16$ & $2.45 \pm 0.07$ & $2.78 \pm 0.05$ \\
$\mathrm{CH}_{4}$ & 1.00 & $0.90 \pm 0.02$ & 3.01 & $3.17 \pm 0.07$ & $2.63 \pm 0.20$ & $2.90 \pm 0.33$ \\
\hline $\mathrm{C}_{3} \mathrm{H}_{8}$ & 0.80 & $0.89 \pm 0.02$ & 2.01 & $2.17 \pm 0.17$ & $2.31 \pm 0.01$ & $2.61 \pm 0.15$ \\
$\mathrm{C}_{3} \mathrm{H}_{8}$ & 0.90 & $0.89 \pm 0.02$ & 2.29 & $2.79 \pm 0.04$ & $2.52 \pm 0.08$ & $2.79 \pm 0.04$ \\
$\mathrm{C}_{3} \mathrm{H}_{8}$ & 1.00 & $0.89 \pm 0.02$ & 2.50 & $2.78 \pm 0.24$ & $2.74 \pm 0.10$ & $2.96 \pm 0.14$ \\
\hline $\mathrm{C}_{2} \mathrm{H}_{4}$ & 0.70 & $1.49 \pm 0.00$ & 1.90 & $2.31 \pm 0.01$ & $2.40 \pm 0.03$ & $2.75 \pm 0.07$ \\
$\mathrm{C}_{2} \mathrm{H}_{4}$ & 0.80 & $1.49 \pm 0.04$ & 2.25 & $2.48 \pm 0.12$ & $2.38 \pm 0.02$ & $2.98 \pm 0.06$ \\
\hline
\end{tabular}




\section{List of figures}

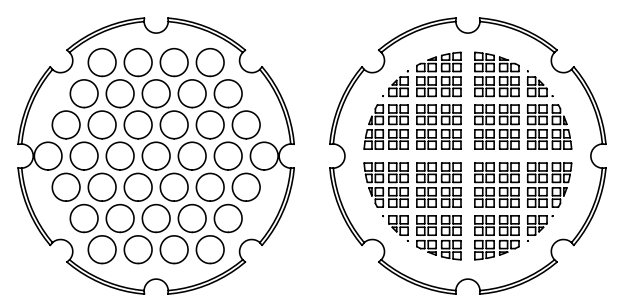

Figure 1: Left: Traditional perforated plates at a blockage ratio of 45\%. Right: Dimensions of the fractal cross grid used in the current study. The dimensions correspond to Grid I used in the study by Geipel et al. [6] and provides a blockage ratio of $65 \%$ with maximum and minimum fractal bar widths of $2.0 \mathrm{~mm}$ and $0.5 \mathrm{~mm}$. 


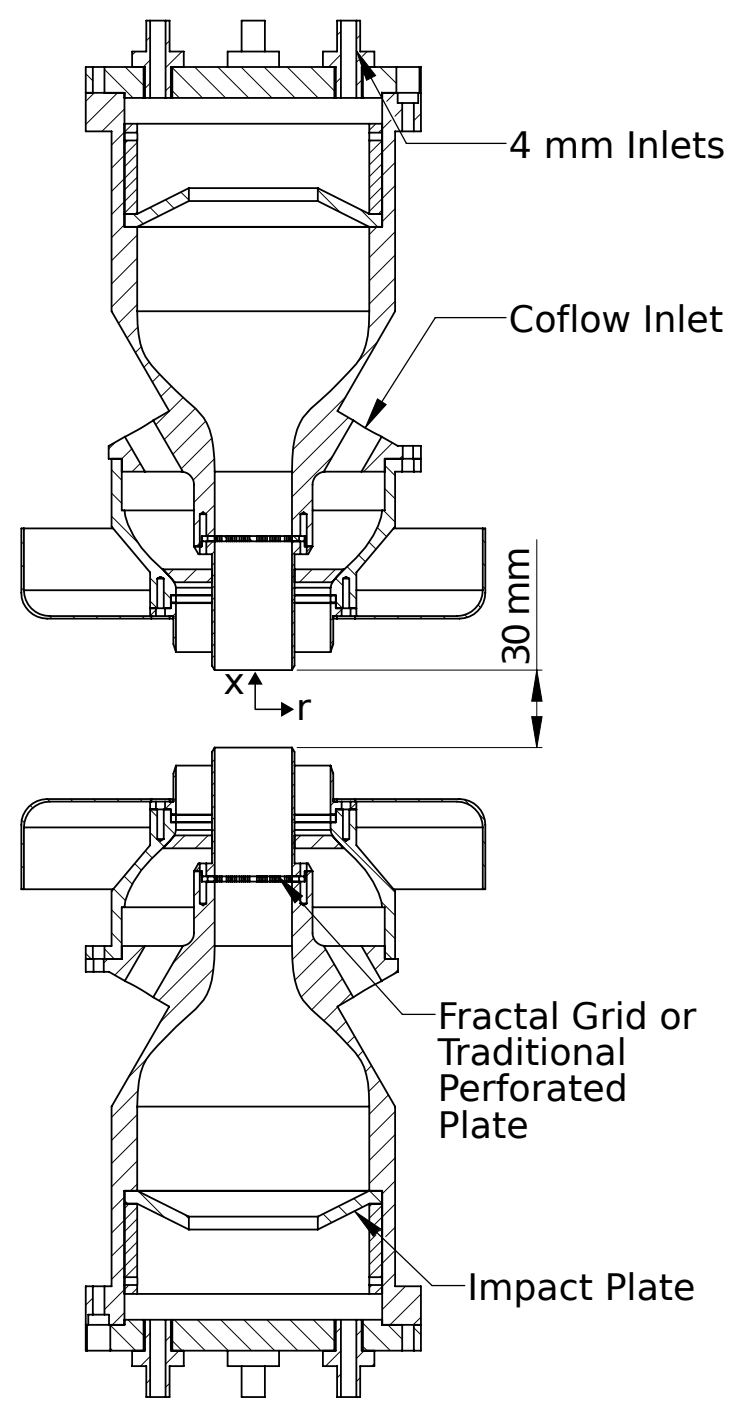

Figure 2: Opposed jet burner geometry showing the locations of the turbulence generators and impact plates. The current geometry is configured for four $4 \mathrm{~mm}$ inlet jets. Alternatively, four $8 \mathrm{~mm}$ inlet jets were also used. 


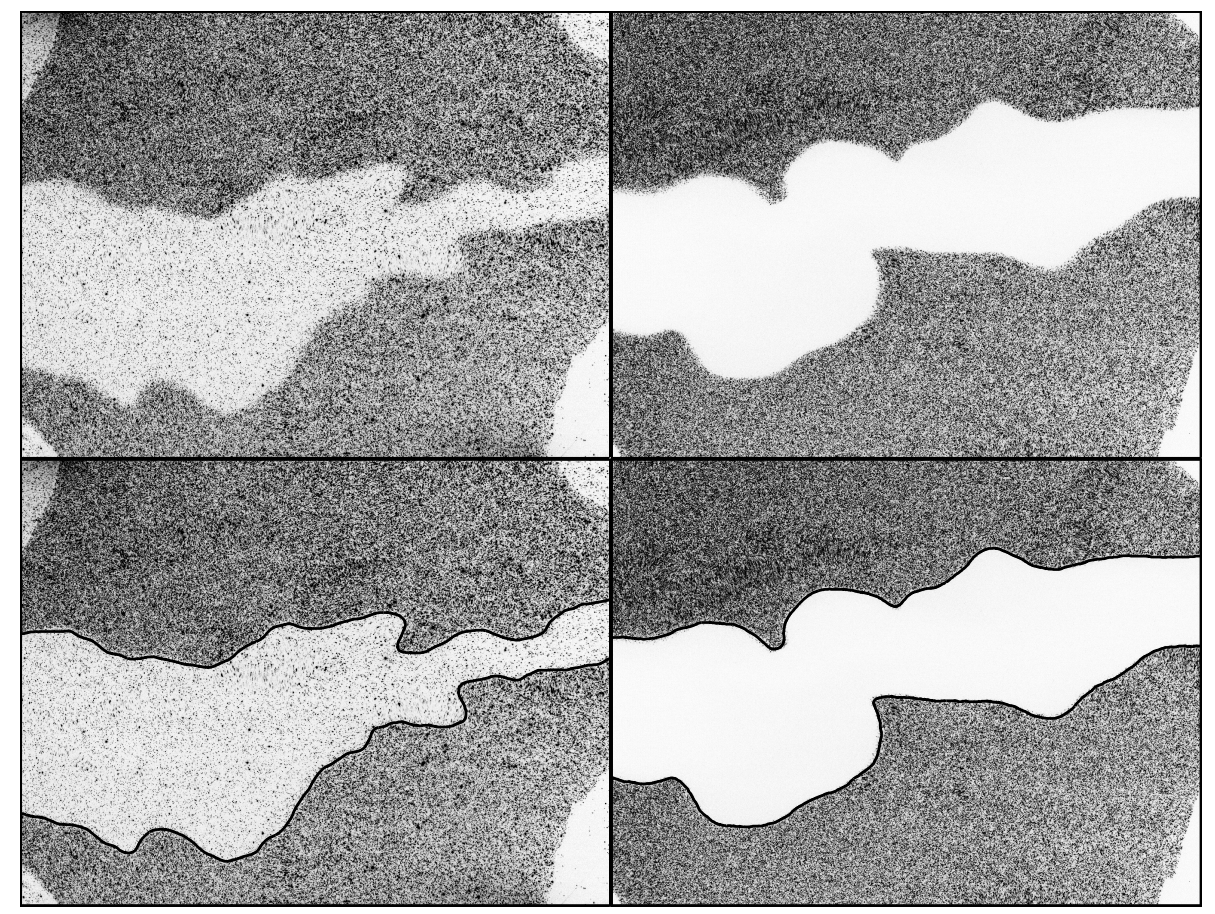

Figure 3: Verification of the flame front detection algorithm using aluminium oxide seeding (left) and silicon oil droplets (right). Lines indicate detected flame fronts.
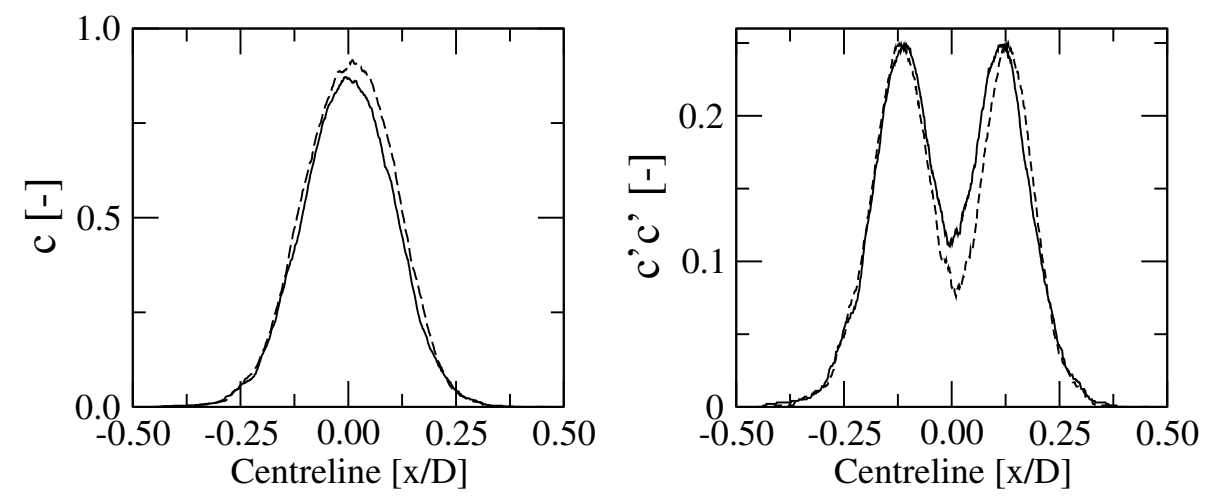

Figure 4: A comparison of centreline results for the first and second moments of the reaction progress variable $(\bar{c})$ obtained using the flame front detection algorithm in combination with aluminium oxide and silicon oil seeding. Solid line: Aluminium oxide seeding. Dashed line: Silicon oil seeding. 

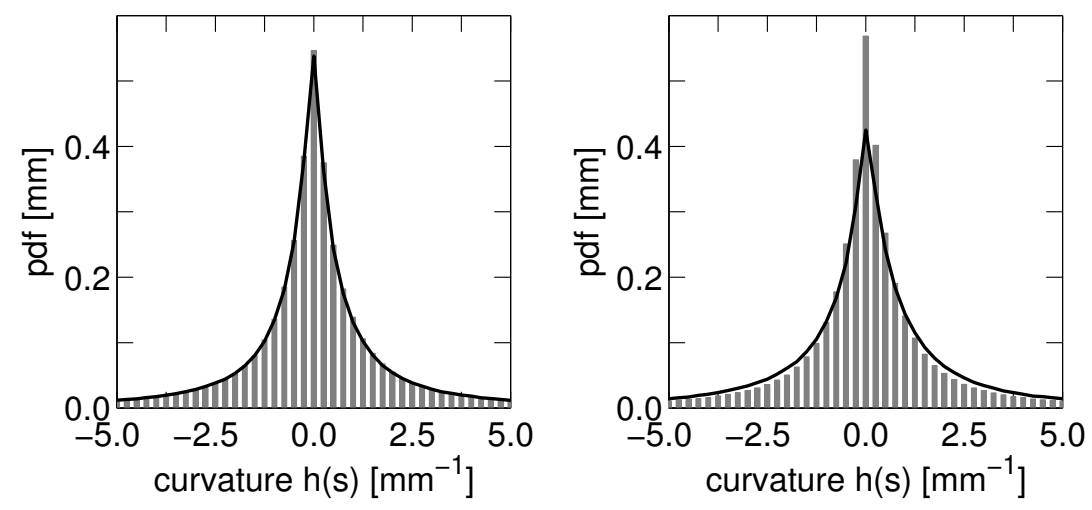

Figure 5: The pdf of curvature. Bars: Aluminium oxide seeding. Lines: Silicon oil seeding. Left: Upper flame front. Right: Lower flame front. 

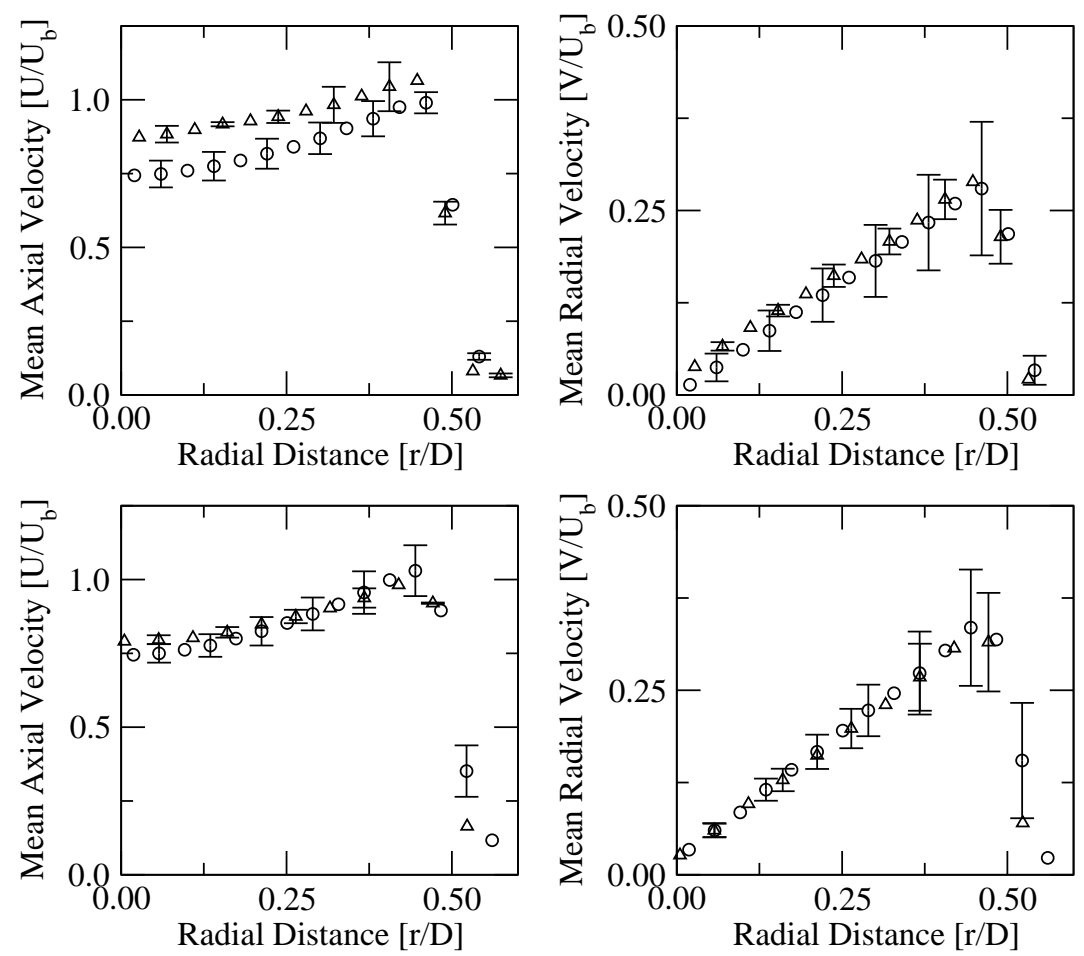

Figure 6: Axial and radial mean velocity profiles plotted across the nozzle radius $1.5 \mathrm{~mm}$ downstream of the nozzle exit. Upper row: $4 \mathrm{~mm}$ jets and a bulk velocity of $4.0 \mathrm{~m} / \mathrm{s}$. Lower row: $8 \mathrm{~mm}$ jets and a average bulk velocity of $4.0 \mathrm{~m} / \mathrm{s}$. Symbols: $\circ$ indicate the use of impact plates and $\triangle$ no impact plates, used in combination with traditional perforated plates. 

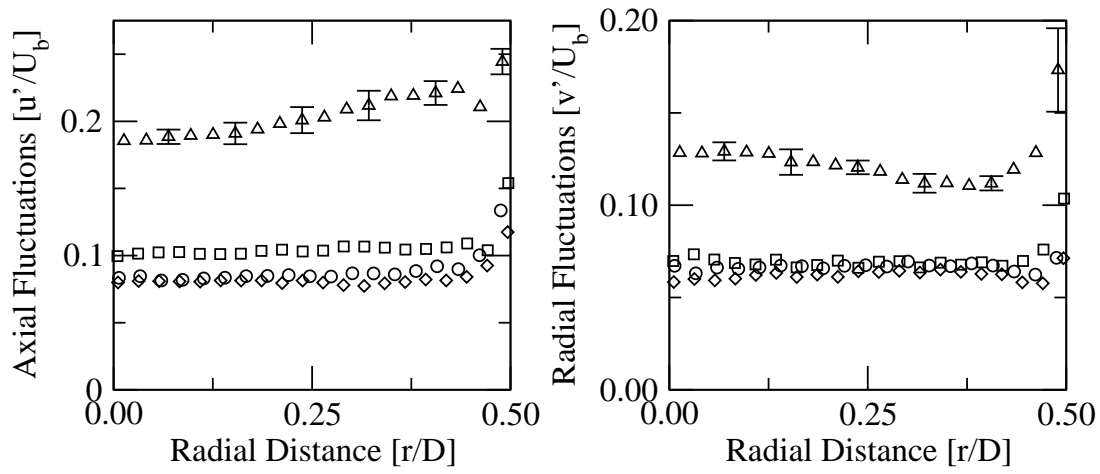

Figure 7: Influence of the upstream boundary conditions on the velocity fluctuations at a mean bulk velocity of $4.0 \mathrm{~m} / \mathrm{s}$. Symbols: $\triangle$ indicates $4 \mathrm{~mm}$ jets and $\square 8 \mathrm{~mm}$ jets measured without impact plates; $\circ$ indicates $4 \mathrm{~mm}$ jets and $\diamond 8 \mathrm{~mm}$ jets with the use of additional impact plates, used in combination with traditional perforated plates. 

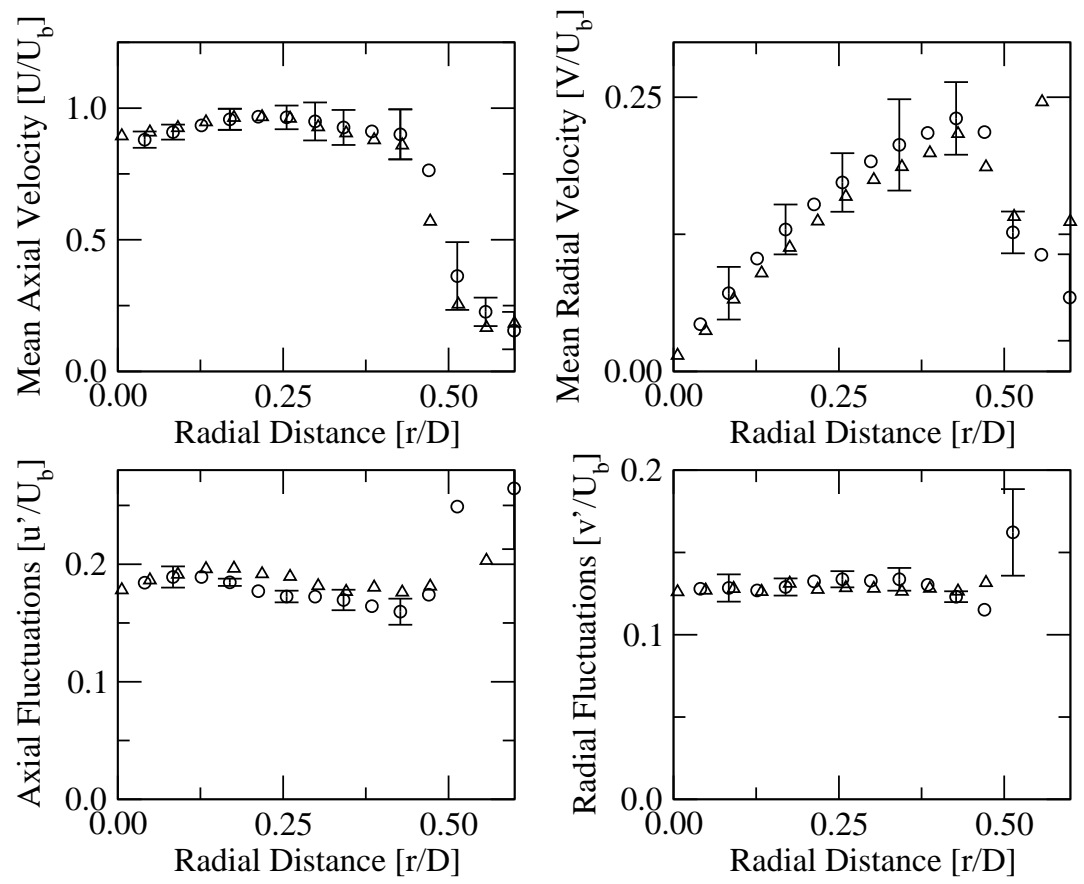

Figure 8: Top row: axial (left) and radial (right) mean velocity profiles measured across the nozzle radius at a location $1.5 \mathrm{~mm}$ downstream of the nozzle exit for a bulk velocity of $4.0 \mathrm{~m} / \mathrm{s}$. Lower row: normalised axial (left) and radial (right) fluctuations; $\circ$ indicates the use of impact plates and $\triangle$ without impact plates. In both cases four $4 \mathrm{~mm}$ inlet jets were used in combination with fractal grids. 

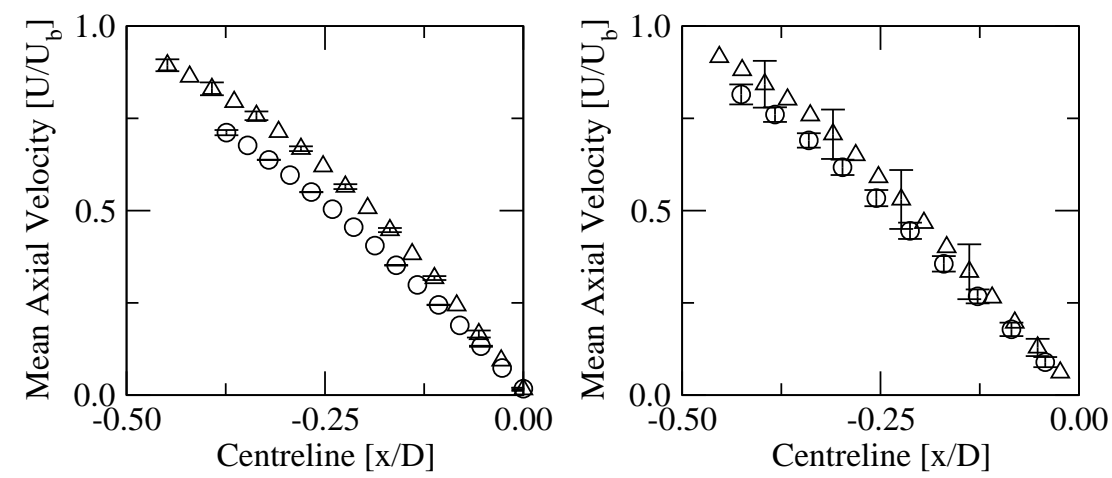

Figure 9: Mean normalised axial velocity profiles along the burner centreline for a bulk velocity of $4.0 \mathrm{~m} / \mathrm{s}$ and using $4 \mathrm{~mm}$ inlet jets with zero indicating the stagnation point. Symbols: $\triangle$ indicates results obtained without impact plates and $\circ$ with impact plates. Left: traditional perforated plates; Right: fractal grids. 

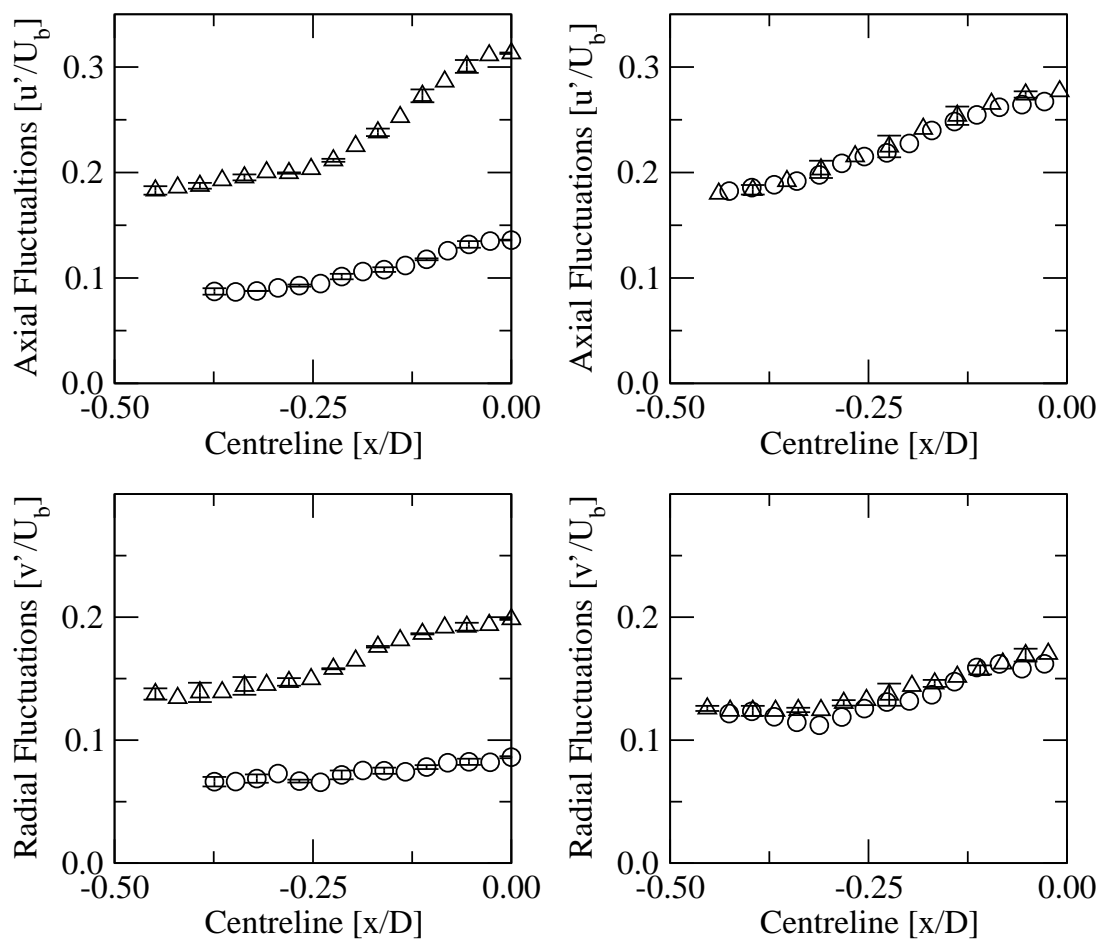

Figure 10: Normalised axial and radial velocity fluctuations along the burner centreline for a bulk velocity of $4.0 \mathrm{~m} / \mathrm{s}$ and with $4 \mathrm{~mm}$ inlet jets with zero indicating the stagnation point. Symbols: $\triangle$ indicates results obtained without impact plates and $\circ$ with impact plates. Left: Traditional perforated plates; Right: fractal grids. 

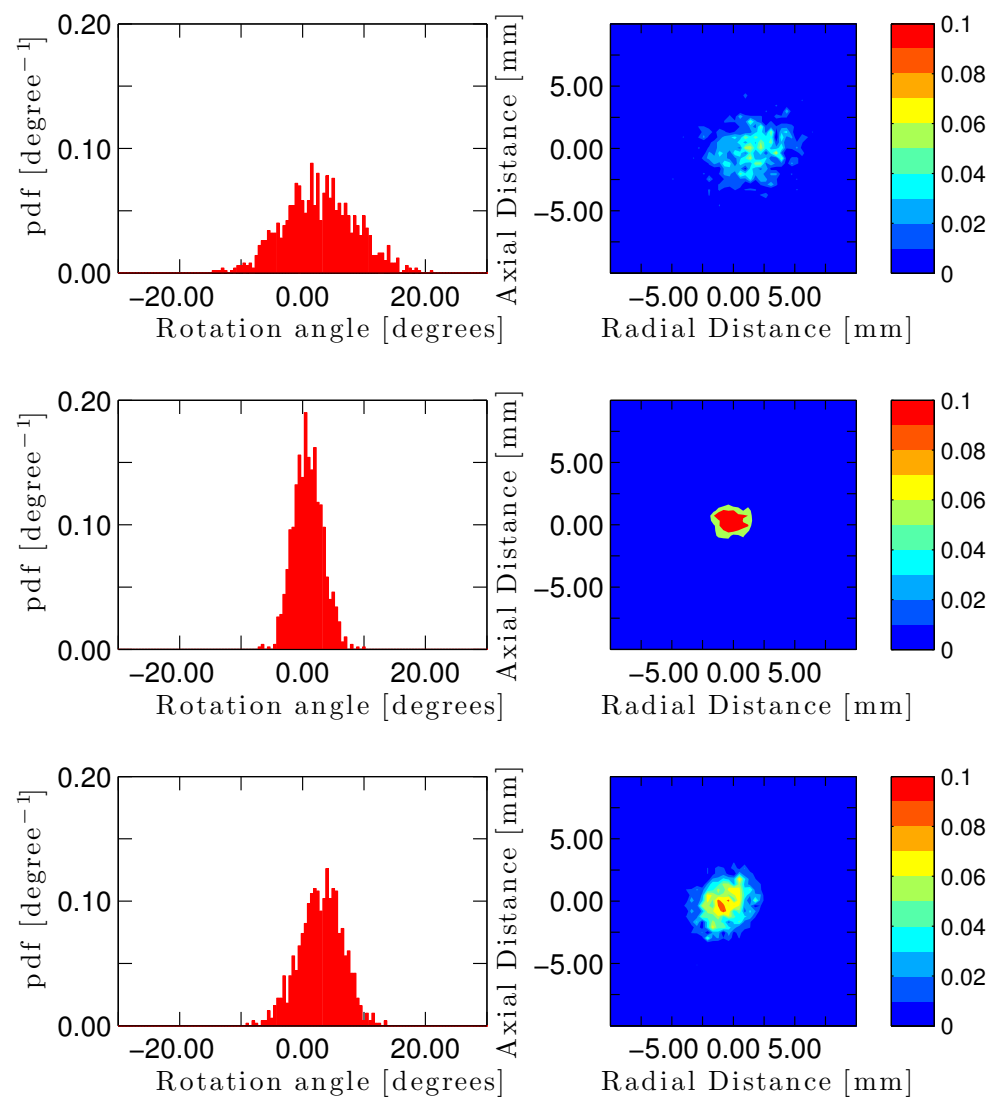

Figure 11: Left: Rotation of the instantaneous stagnation plane. Right: Probability density functions (pdfs in $\mathrm{mm}^{-2}$ ) of the instantaneous stagnation point location. Methane flames with $\phi=0.9$ and a bulk velocity of $4.0 \mathrm{~m} / \mathrm{s}$. Top: Perforated plates without impact plates. Middle: perforated plates with impact plates. Bottom: Fractal grids with impact plates. 

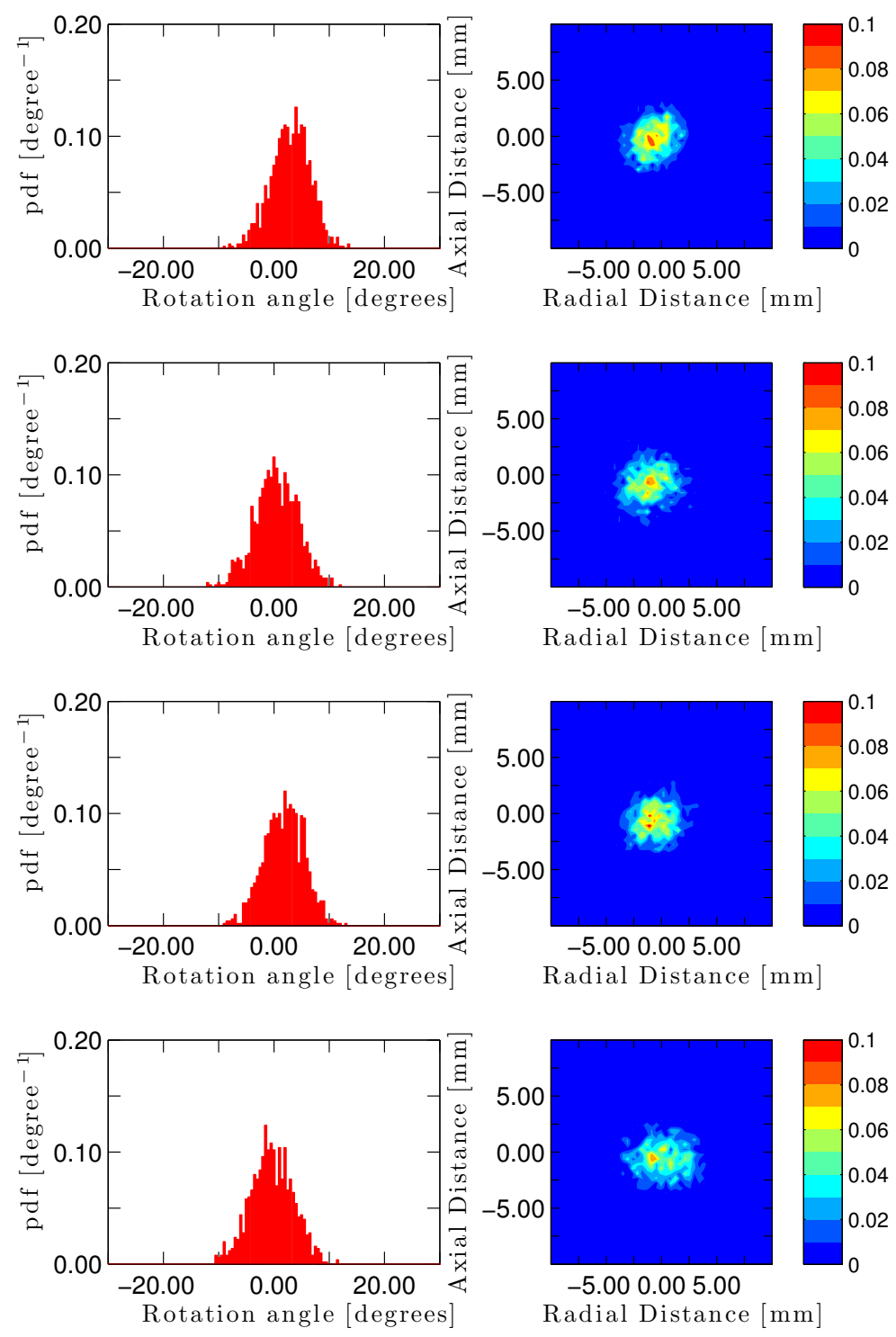

Figure 12: Left: Rotation of the instantaneous stagnation plane. Right: Probability density functions (pdfs in $\mathrm{mm}^{-2}$ ) of the instantaneous stagnation point location. Methane flames with $\phi=0.9$ and a bulk velocity of $4.0 \mathrm{~m} / \mathrm{s}$ measured at four circumferential positions. First and third row are measured at $45^{\circ}$ and $135^{\circ}$ rotation with respect to the largest fractal cross. The second and last rows are measured along the two perpendicular parts of the largest fractal cross shown in Fig. 1. 


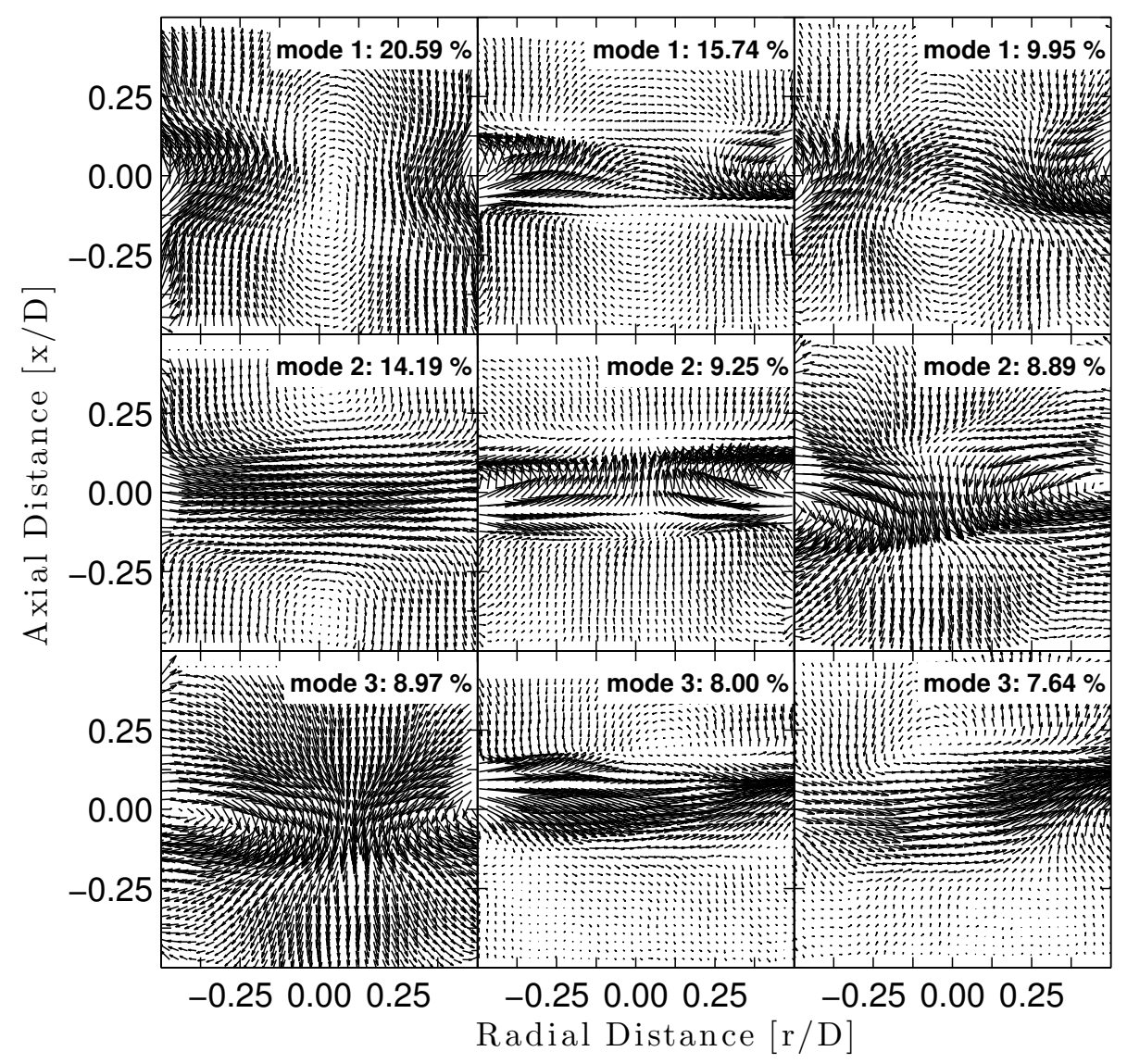

Figure 13: Comparison of the first three eigenmodes for premixed methane flames with $\phi$ $=0.9$ and a bulk velocity of $4.0 \mathrm{~m} / \mathrm{s}$. Left column: Perforated plates with no impact plates. First eigenmode with $20.6 \%$, second eigenmode with $14.2 \%$ and third eigenmode with $9.0 \%$ of the turbulent kinetic energy. Middle column: Perforated plates with additional impact plates. First eigenmode with $15.7 \%$, second eigenmode with $9.3 \%$ and third eigenmode with $8.0 \%$ of the kinetic energy. Right column: Fractal grids with additional impact plates. First eigenmode with $10.0 \%$, second eigenmode with $8.9 \%$ and third eigenmode with $7.6 \%$ of the kinetic energy. 

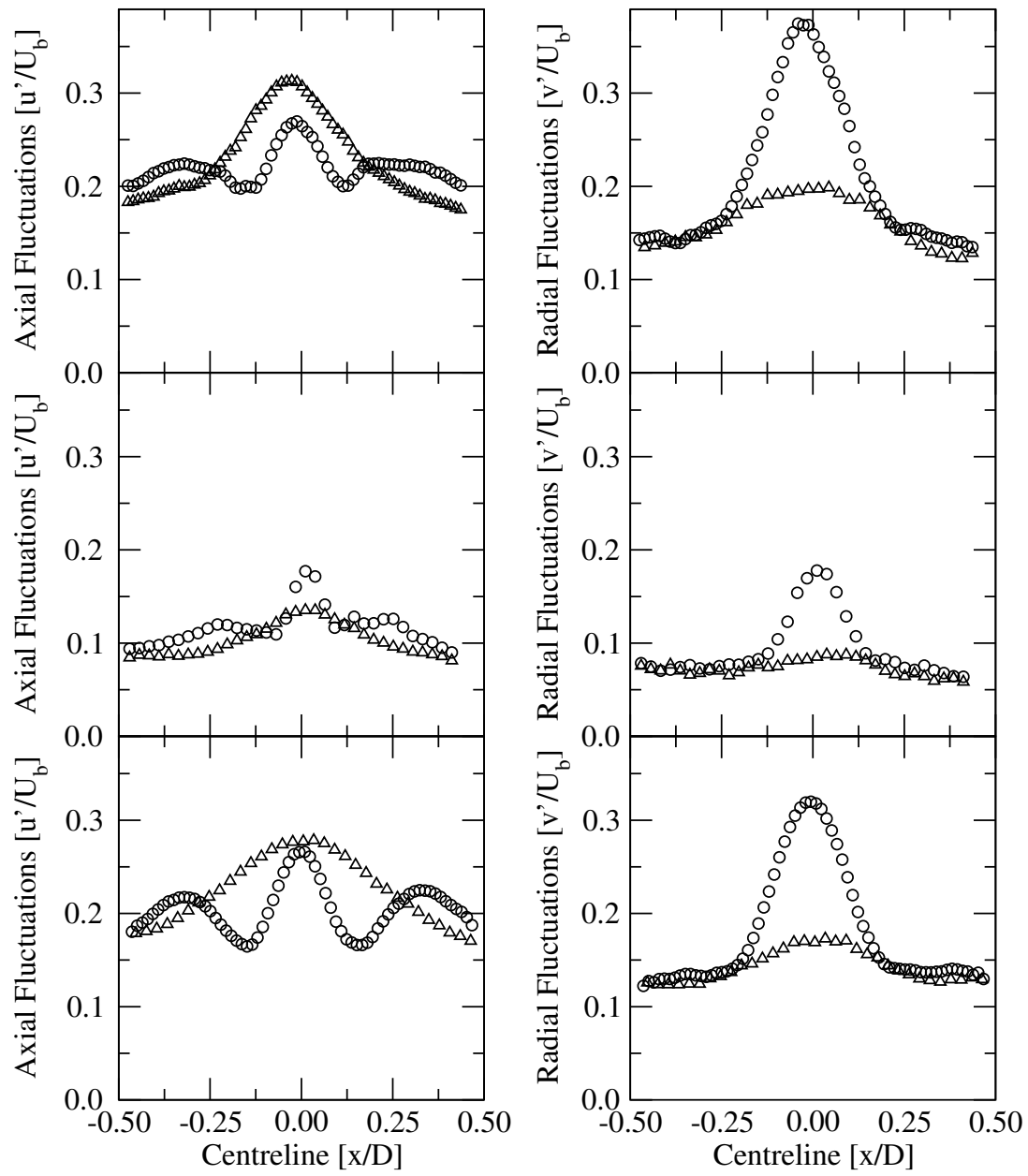

Figure 14: Comparison of velocity fluctuations for isothermal $(\triangle)$ and reacting $(\circ)$ flows along the burner centreline for methane/air flames with $\phi=0.9$ and a bulk velocity of $4.0 \mathrm{~m} / \mathrm{s}$. Top: Perforated plates without impact plates. Middle: Perforated plates with impact plates. Bottom: Fractal grids with impact plates. 

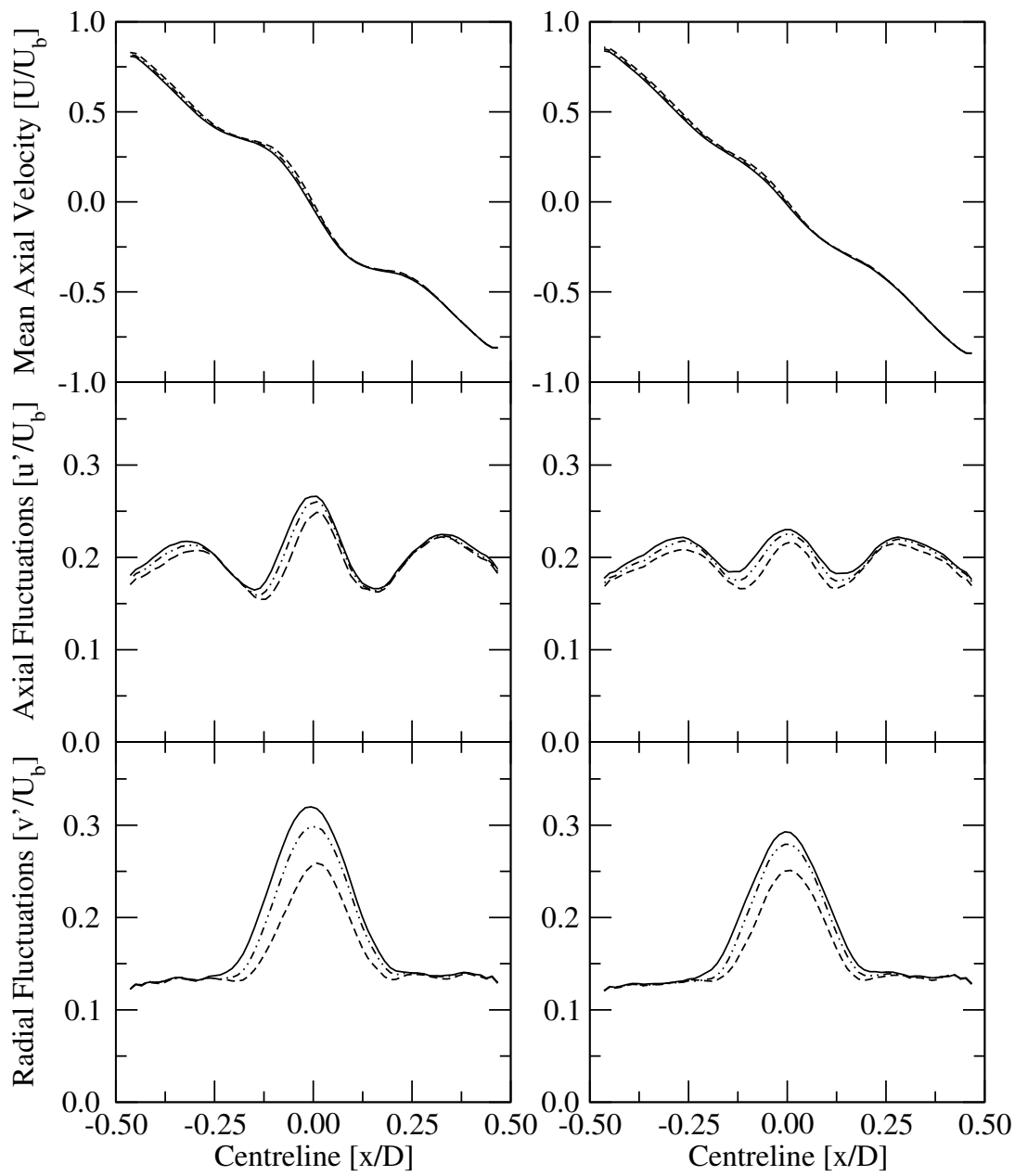

Figure 15: Effect of an increasing a circular filtering threshold on centreline profiles of the normalised axial mean velocity (upper), axial (middle) and radial (lower) velocity fluctuations for a methane/air flame with $\phi=0.8$ (right) and $\phi=0.9$ (left) at a bulk velocity of $4.0 \mathrm{~m} / \mathrm{s}$. Solid line: No threshold. Dashed-dotted line filtering with a $3.5 \mathrm{~mm}$ threshold. Dashed line filtering with a $2.5 \mathrm{~mm}$ threshold. All profiles were obtained using fractal grids with fitted impact plates. 

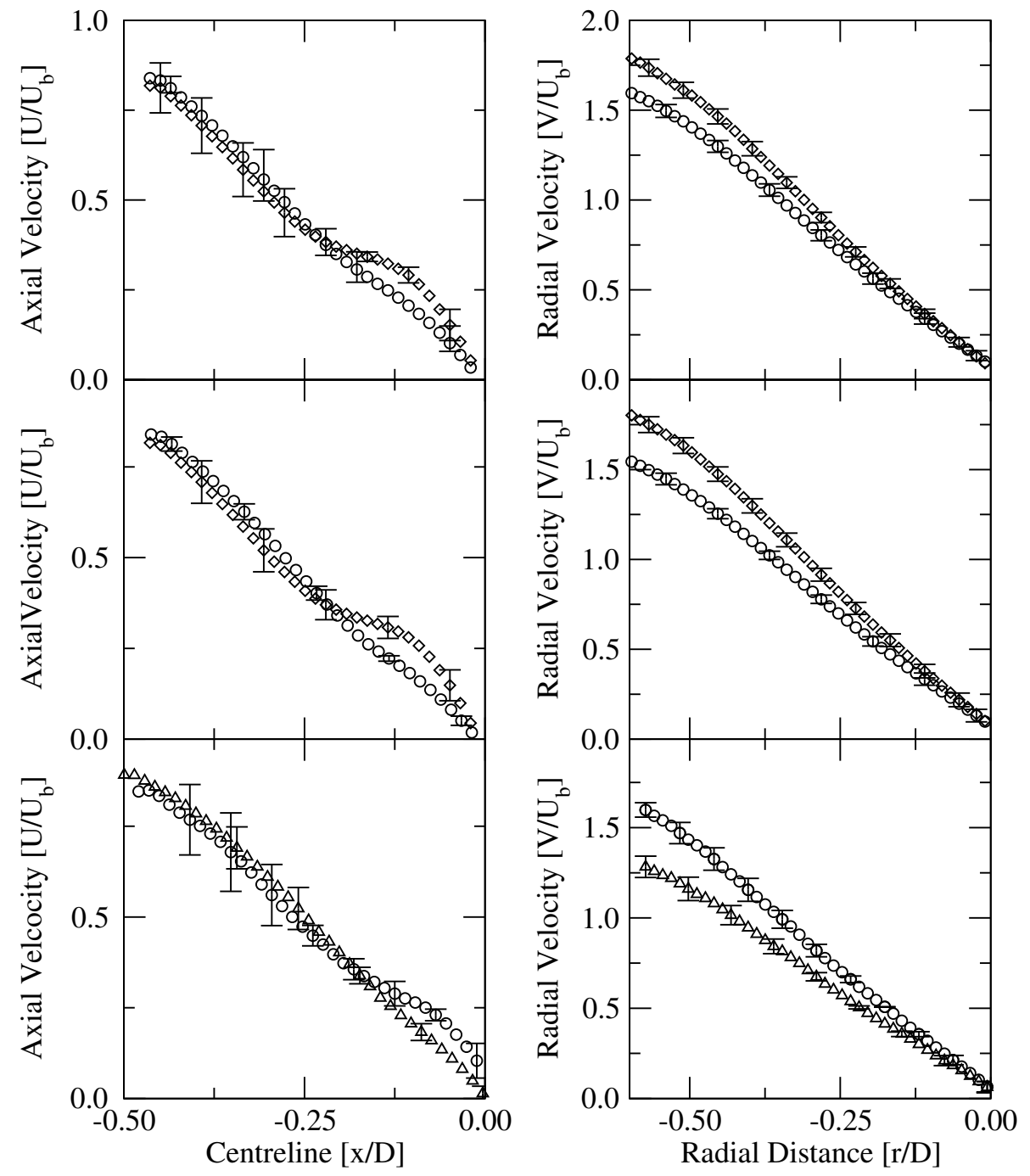

Figure 16: Mean normalised axial velocities (left) along the burner centreline and mean normalised radial velocities (right) at the stagnation plane for methane (top) and propane (middle) flames at a bulk velocity of $4.0 \mathrm{~m} / \mathrm{s}$. Ethylene flames (bottom) are shown for a bulk velocity of $8.0 \mathrm{~m} / \mathrm{s}$. Equivalence ratios of $\diamond=0.9, \circ=0.8$ and $\triangle=0.7$. 

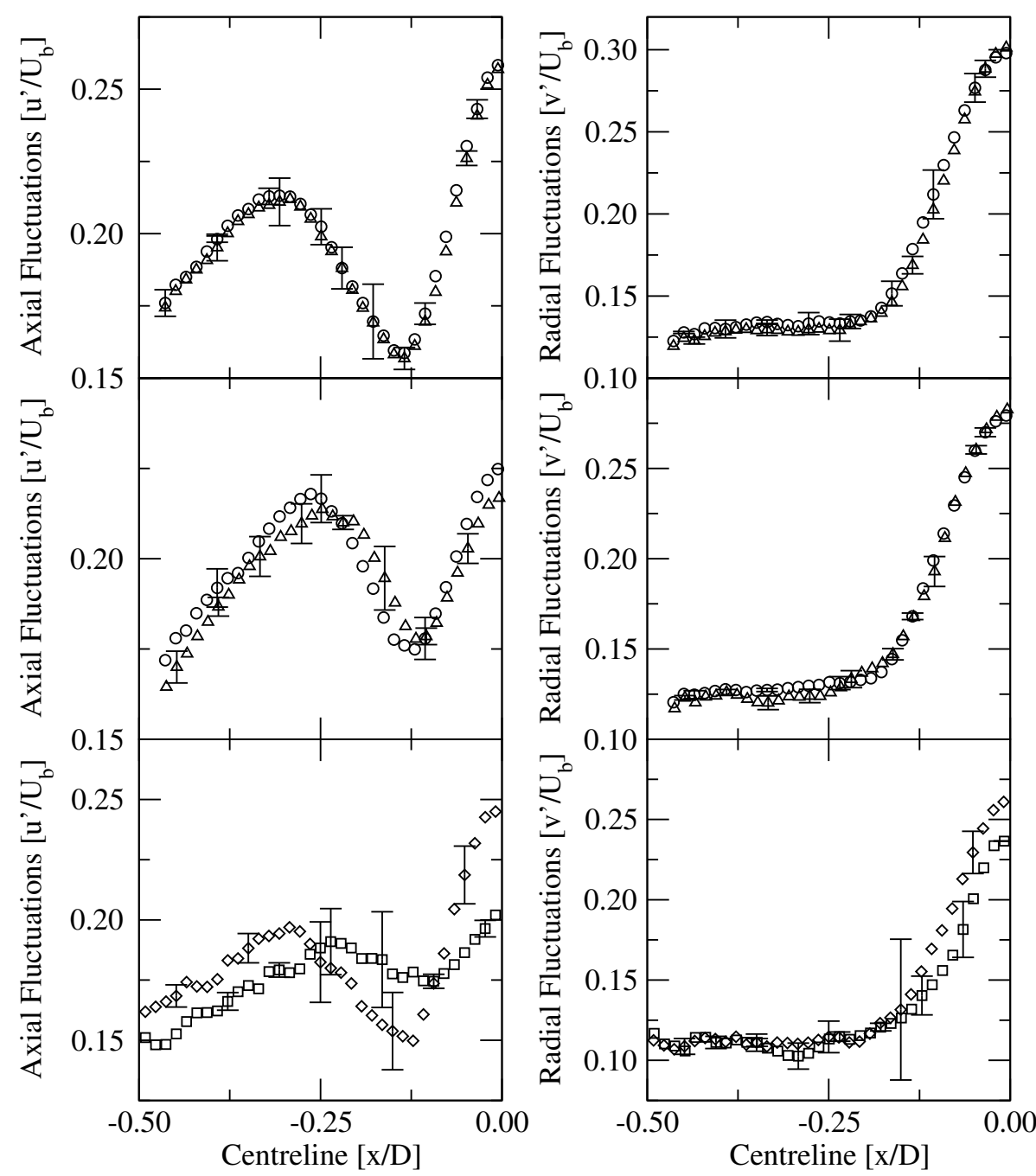

Figure 17: Comparison of the normalised axial (left) and radial (right) velocity fluctuations along the burner axis for methane $(\circ)$ and propane $(\triangle)$ flames at a bulk velocity of $4.0 \mathrm{~m} / \mathrm{s}$. Top row: $\phi=0.9$. Middle row $\phi=0.8$. Bottom row: Normalised axial (left) and radial (right) velocity fluctuations along the burner axis for ethylene flames at $\phi=0.8(\diamond)$ and $\phi=0.7(\square)$ at $8.0 \mathrm{~m} / \mathrm{s}$. 

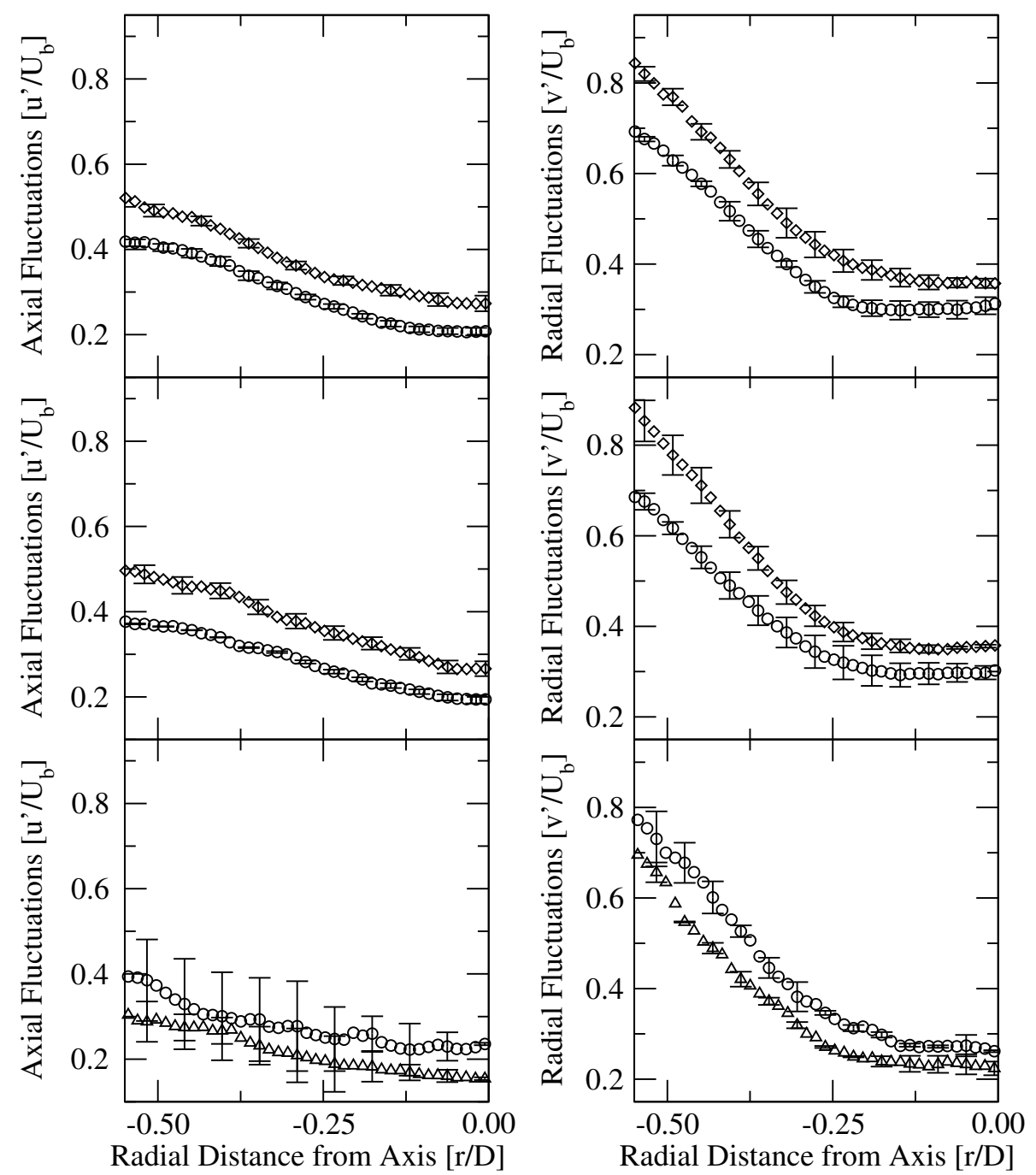

Figure 18: Normalised axial (left) and radial (right) velocity fluctuations for methane (top) and propane (middle) flames along the stagnation plane for a $4.0 \mathrm{~m} / \mathrm{s}$ bulk velocity. Results for ethylene flames (bottom) were obtained for a bulk velocity of $8.0 \mathrm{~m} / \mathrm{s}$. Stoichiometries of $\diamond=0.9, \circ=0.8$ and $\triangle=0.7$. 

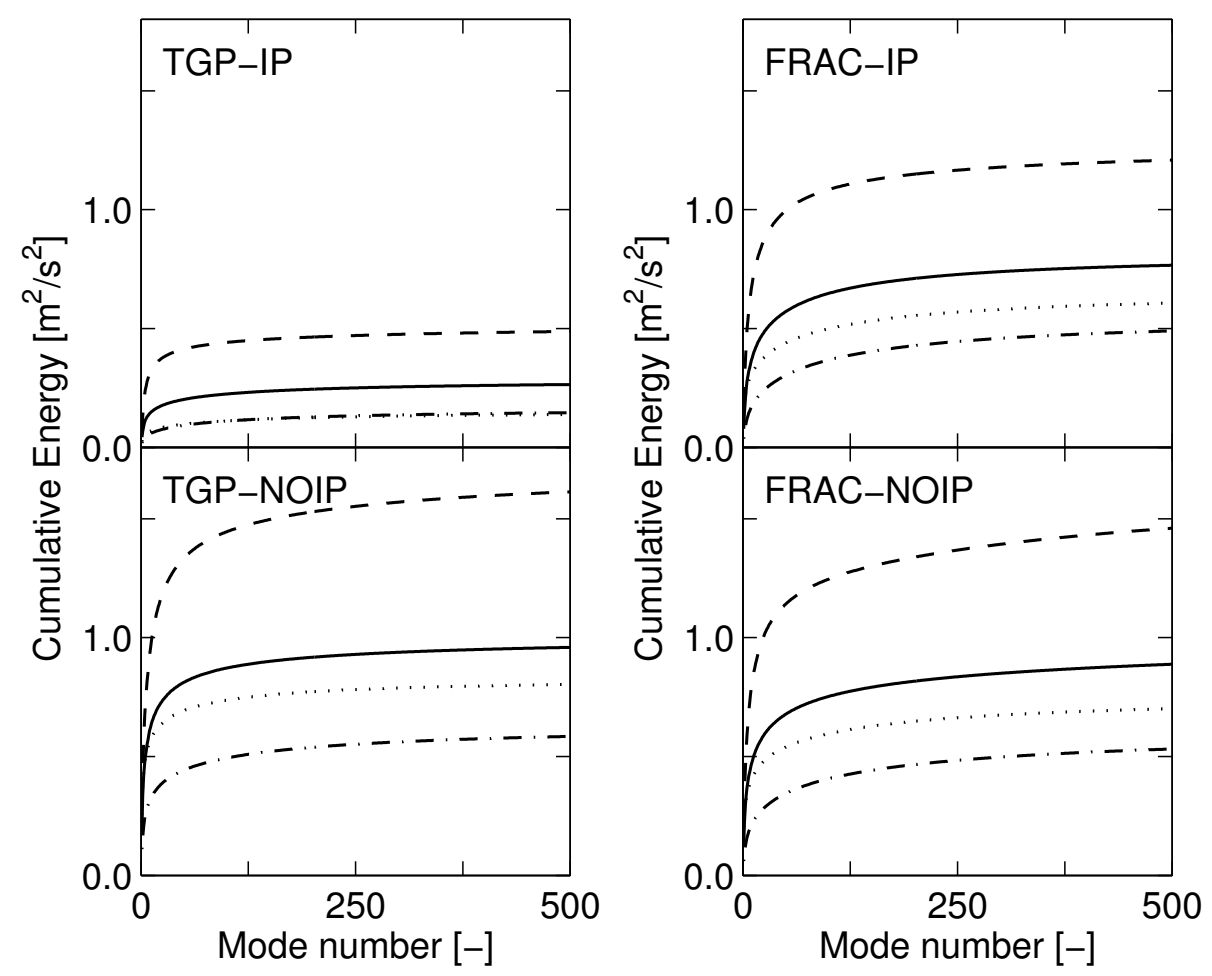

Figure 19: Cumulative energy in modes via conditional and unconditional POD, normalised and multiplied by the mean turbulence energy in the flow field for methane-air flames with $\phi=0.90$, averaged over all images in each set. The turbulence energy was for comparison purposes defined to be $\left(\frac{1}{2}\right)\left(u^{\prime} u^{\prime}+v^{\prime} v^{\prime}\right)$. Conventional turbulence generating plates with (TGP-IP) and without (TGP-NOIP) fitted impact plate. Fractal grid with (FRAC-IP) and without (FRAC-NOIP) fitted impact plate. Dotted line: Isothermal flow; Dashed-dotted line: Reactants; Dashed Line: Products; Solid line: Unconditional POD. 

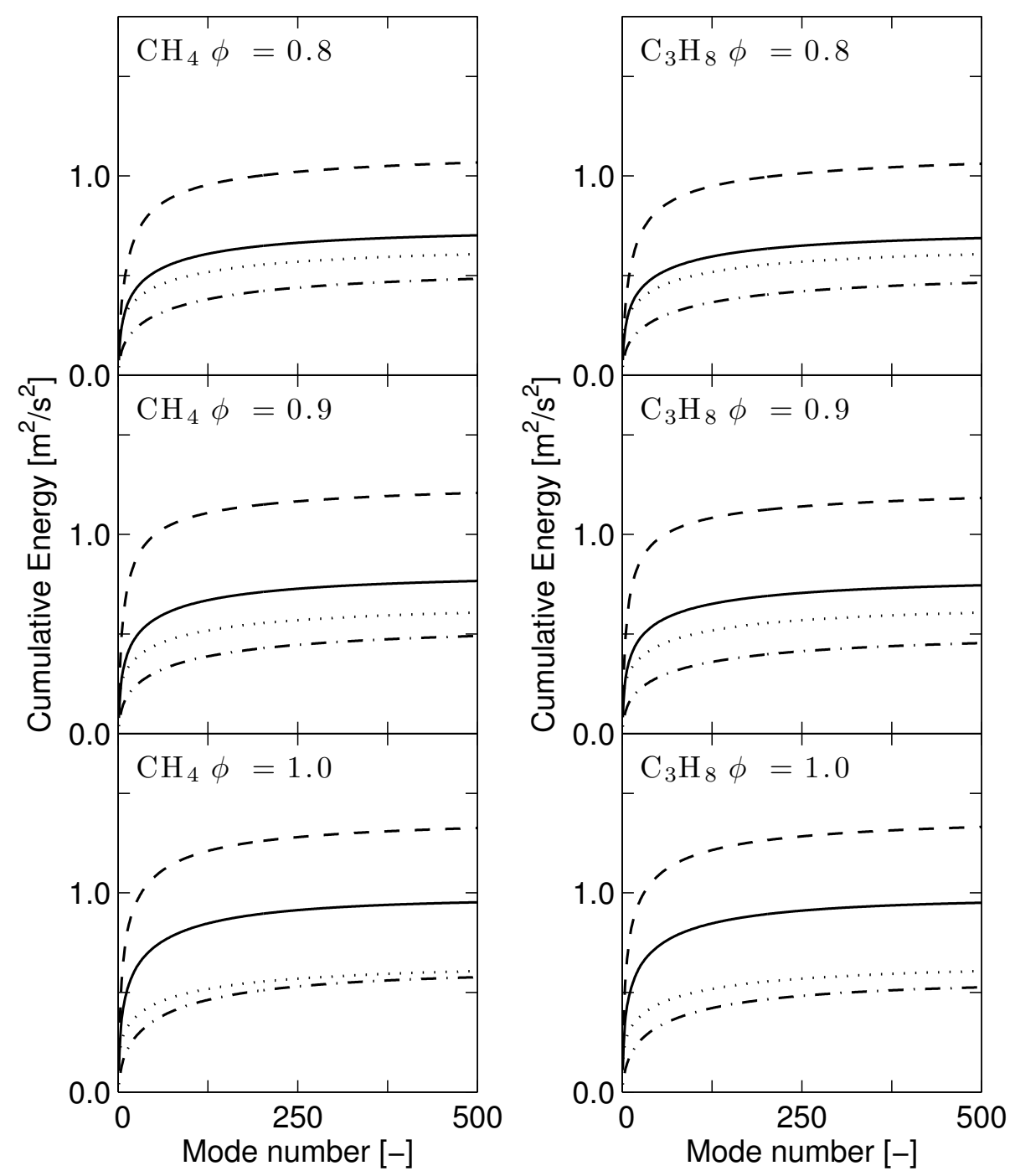

Figure 20: Cumulative energy in modes via conditional and unconditional POD, normalised and multiplied by the mean turbulence energy in the flow field averaged over all images in each set. The turbulence energy was for comparison purposes defined to be $\left(\frac{1}{2}\right)\left(u^{\prime} u^{\prime}+v^{\prime} v^{\prime}\right)$. Fractal grids with a fitted impact plate used in all cases. Left column: Methane-air flame with $\phi=0.80$ (top), 0.90 (middle) and 1.0 (bottom). Right column: Propane-air flame with $\phi=0.80$ (top), 0.90 (middle) and 1.0 (bottom). Dotted line: Isothermal flow; Dashed-dotted line: Reactants; Dashed Line: Products; Solid line: Unconditional POD. 

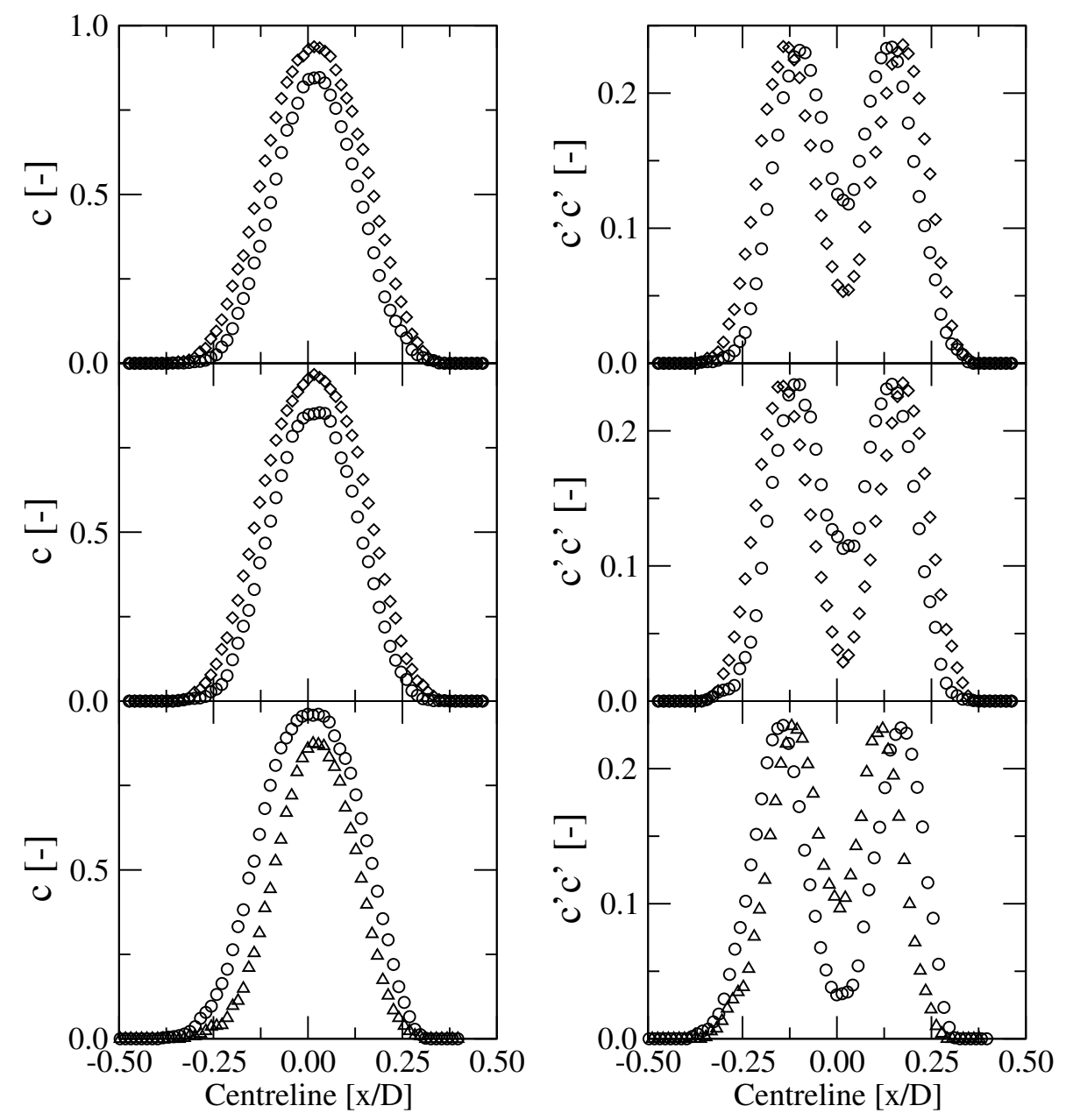

Figure 21: Comparisons of the mean progress variable $(\bar{c})$ (left) and its variance $\left(\overline{c^{\prime} c^{\prime}}\right)$ (right) for methane (top) and propane (middle) flames with a $4.0 \mathrm{~m} / \mathrm{s}$ bulk velocity. Ethylene flames (bottom) at a bulk velocity of $8.0 \mathrm{~m} / \mathrm{s}$. Stoichiometries of $\phi=0.9(\diamond), \phi$ $=0.8(\circ)$ and $\phi=0.7(\triangle)$. 

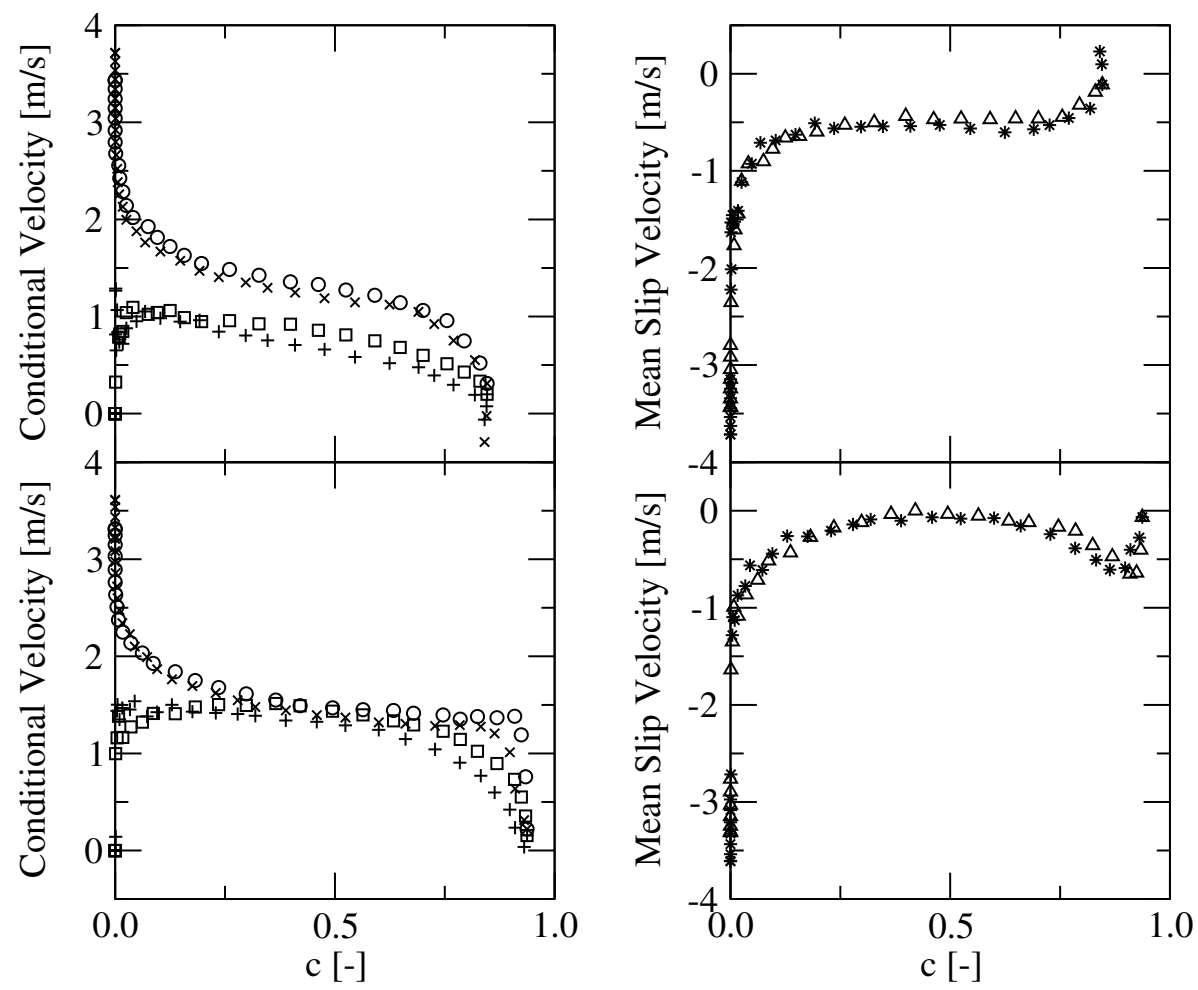

Figure 22: Conditional reactant and product velocities (left) and slip velocities (right) for methane flames. (०) Reactant $\left(\bar{u}_{r}\right)$ and $(\square)$ product $\left(\bar{u}_{p}\right)$ velocities for the upper nozzle. $(\times)$ Reactant $\left(\bar{u}_{r}\right)$ and $(+)$ product $\left(\bar{u}_{p}\right)$ velocities for the lower nozzle. Mean slip velocities $\left(u_{s}\right)$ for the upper $(\triangle)$ and lower $(*)$ nozzles. Top row: $\phi=0.8$. Bottom row: $\phi=0.9$. 

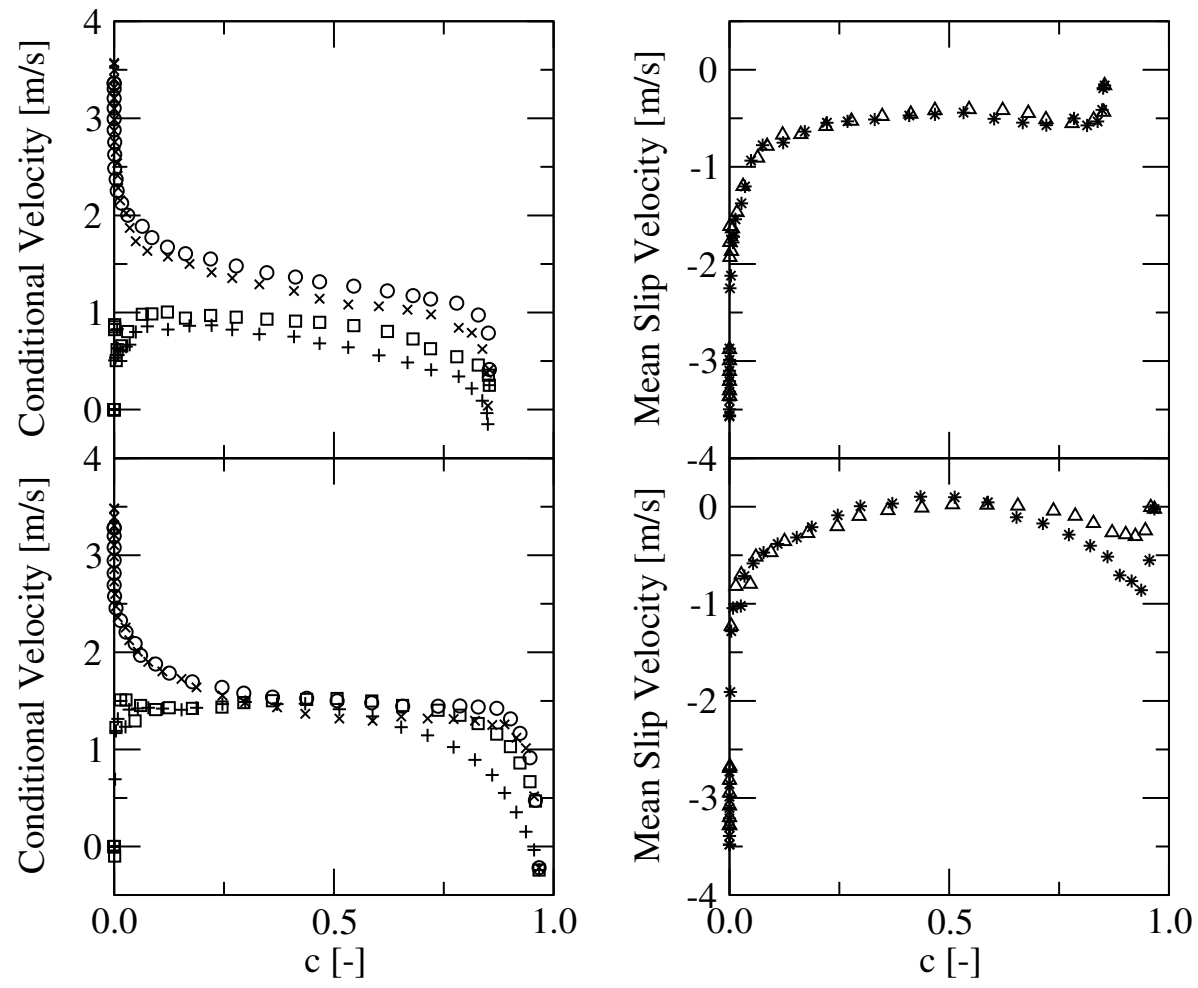

Figure 23: Conditional reactant and product velocities (left) and slip velocities (right) for propane flames. (०) Reactant $\left(\bar{u}_{r}\right)$ and $(\square)$ product $\left(\bar{u}_{p}\right)$ velocities for the upper nozzle. $(\times)$ Reactant $\left(\bar{u}_{r}\right)$ and $(+)$ product $\left(\bar{u}_{p}\right)$ velocities for the lower nozzle. Mean slip velocities $\left(u_{s}\right)$ for the upper $(\triangle)$ and lower $(*)$ nozzles. Top row: $\phi=0.8$. Bottom row: $\phi=0.9$. 


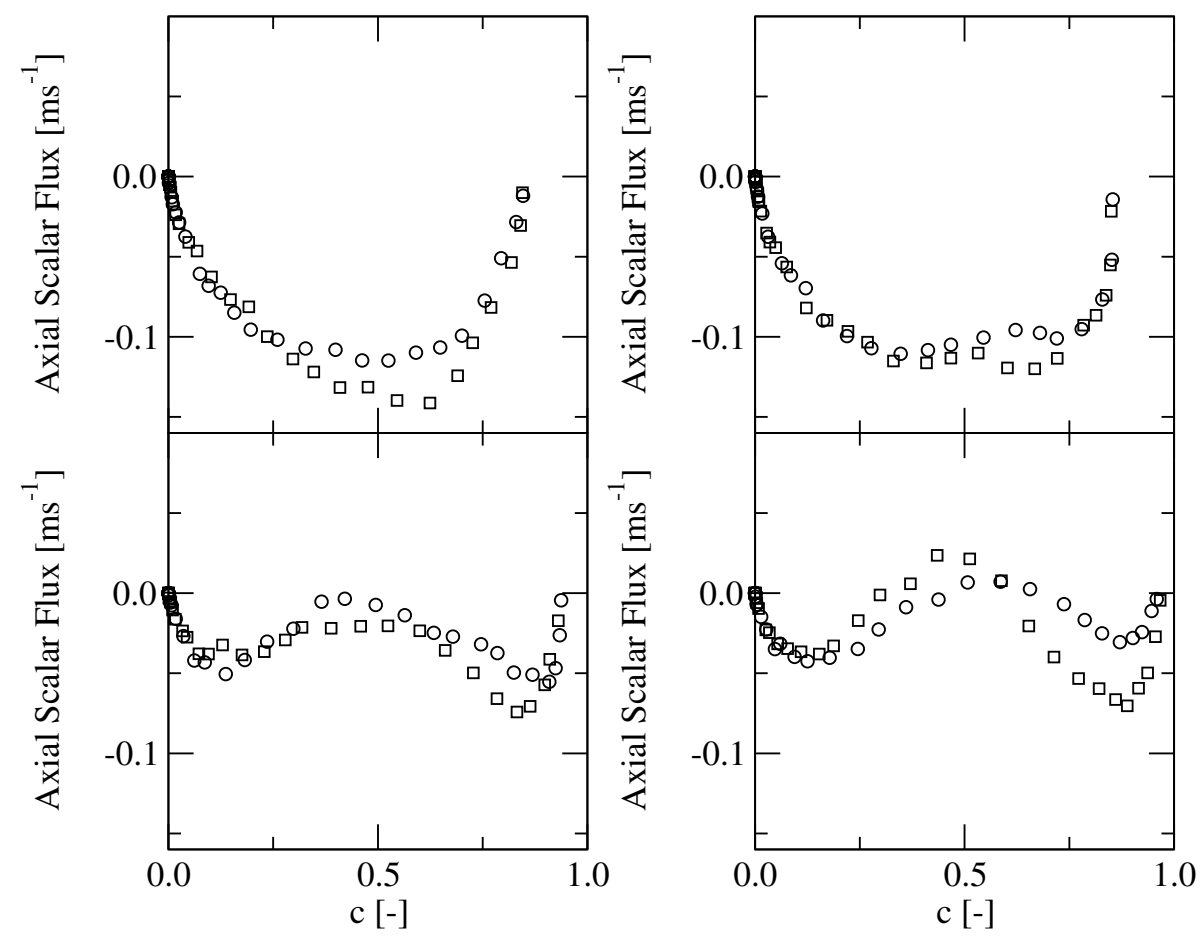

Figure 24: Axial scalar fluxes $\overline{u^{\prime} c^{\prime}}$ for methane (left) and propane (right) flames shown in reaction progress variable $(\bar{c})$ space. o Upper nozzle. $\square$ Lower nozzle. Top row: $\phi=0.8$. Bottom row: $\phi=0.9$. 

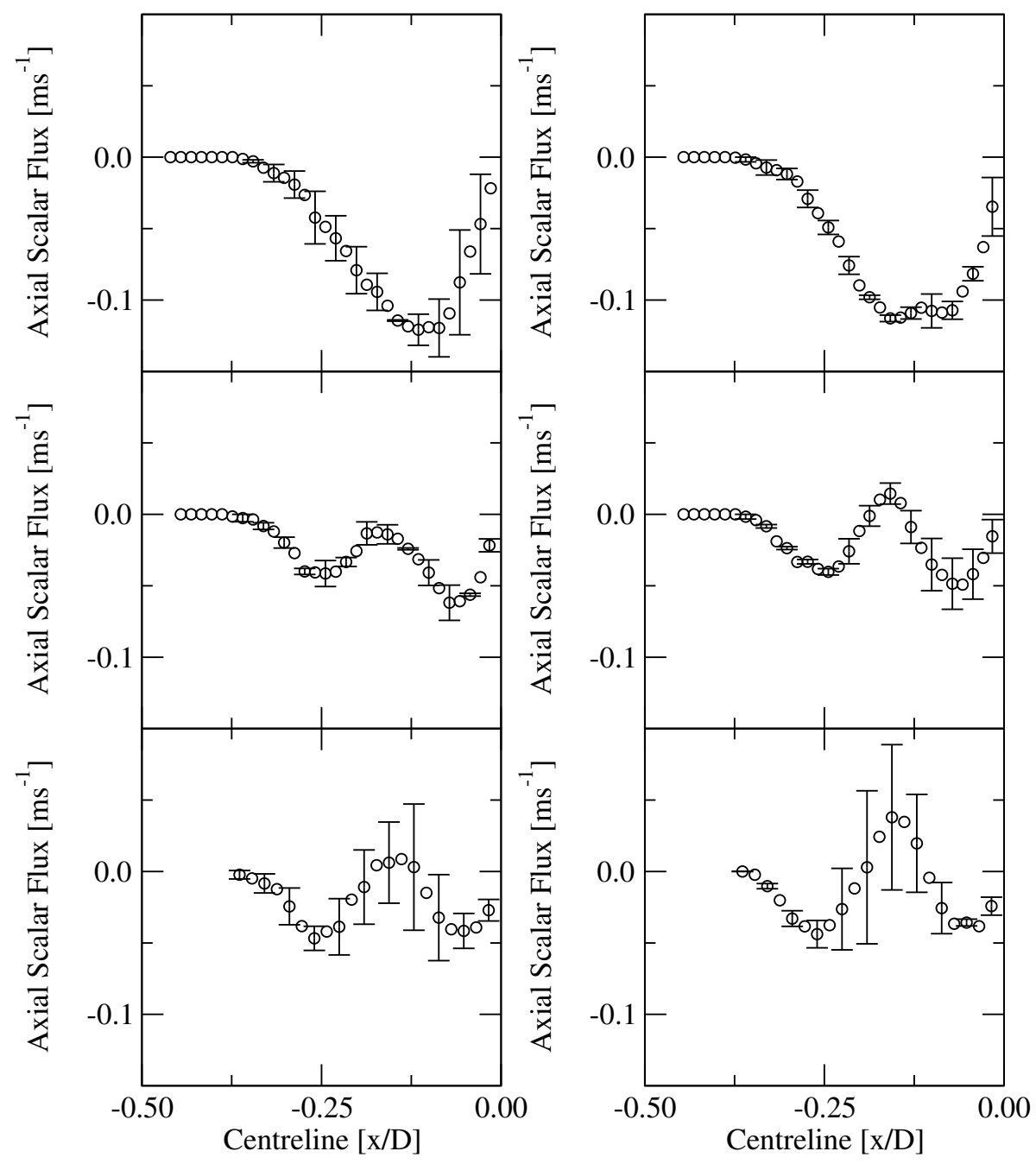

Figure 25: Axial scalar fluxes $\overline{u^{\prime} c^{\prime}}$ for methane (left) and propane (right) flames shown in physical space. Stoichiometries of $\phi=0.8$ (top), $\phi=0.9$ (middle) and $\phi=1.0$ (bottom). 